\title{
Logics without the contraction rule and residuated lattices
}

\author{
Hiroakira Ono \\ Japan Advanced Institute of Science and Technology \\ Nomi, IshikaWa, Japan \\ ono@jaist.ac.jp \\ Received by Greg Restall \\ Published September 22, 2010 \\ http://www.philosophy.unimelb.edu.au/ajl/2010 \\ (C) 20 Io Hiroakira Ono
}

To the memory of R. K. Meyer

\begin{abstract}
In this paper, we will develop an algebraic study of substructural propositional logics over $F L_{e w}$, i.e. the logic which is obtained from the intuitionistic logics by eliminating the contraction rule. Our main technical tool is to use residuated lattices as the algebraic semantics for them. This enables us to study different kinds of nonclassical logics, including intermediate logics, BCK-logics, Łukasiewicz's many-valued logics and fuzzy logics, within a uniform framework.
\end{abstract}

FOREWORD (2009): The draft of the present paper was originally completed in 1999 and then revised slightly in $200 \mathrm{I}$, which I intended to dedicate to R. K. Meyer on the occasion of his 65 th birthday.

From the middle of 9os, I had been trying to develop an algebraic study of substructural logics over the logic without the contraction rule $\mathrm{FL}_{\mathrm{ew}}$, and had announced results in several conferences, e.g. the 6th Asian Logic Conference in 1996, Dagstuhl Seminar on Multiple-Valued Logic in 1997 and S. Jaśkowski Memorial Symposium on Parainconsistent Logic, Logical Philosophy, Mathematics \& Informatics in 1998 . So I planned to make the present paper a comprehensive survey of the state of affairs of the study.

While the paper has not been published for many years, the draft has been referred in considerably many papers of substructural logics published in the last decade.

On the occasion of the publication of the paper, I have been wondering in which way I should publish the paper and how much I should revise it. For, there have been a remakable progress in the direction of this research within these Io years, and moreover my joint book [25] on substructural logics was already published. It would be no use to make an entire update of it. 
My decision is to minimize changes, keeping the original form, and to add only necessary information on recent progress. (I put the mark ( $\dagger$ ) to footnotes which are essentially added in the present revision.) I hope that the paper will be still of interest and also informative, especially in its references, beyond its historical meaning.

\section{INTRODUCTION}

A lot of works have been done in recent years for substructural logics, logics lacking some or all of structural rules when they are formalized in sequent calculi (see e.g. [2I]). They include various kinds of nonclassical logics like Lambek calculus for categorial grammar, linear logic, BCK-logic and relevant logics, which have been introduced by different motivations with their own interests and aims and have been studied separately. The study of substructural logics, if successful, will enable us to develop the study of various nonclassical logics in a uniform viewpoint, and to discuss common features among them within this framework.

Though proof-theoretic methods have showed their effectiveness for particular substructural logics, e.g. logics formalized in cut-free sequent systems (see e.g. [54]), it will be quite necessary to introduce semantical methods when we want to develop a general study of substructural logics to the similar extent to that of modal logics.

In this paper, we will develop an algebraic study of substructural logics over $F L_{e w}$. The sequent calculus $F L_{e w}$ is obtained from the intuitionistic logic by deleting the contraction rule. Sometimes we call substructural logics over $\mathrm{FL}_{\mathrm{ew}}$, or logics without contraction rule, although the contraction rule holds in some of them. The class of logics without the contraction rule contains intermediate logics, BCK-logics, Łukasiewicz's many-valued logics and fuzzy logics (in the sense of $[30]$ ). Our main technical tool here is algebraic one which is based on closer connections between logics over $\mathrm{FL}_{e w}$ and classes of residuated lattices.

Our main aims of the present paper are first to view known results on various logics without contraction in our framework, to keep the situation in perspective and to try to find out proper directions of further study of substructural logics over $F L_{e w}$. We give here some basic references of various classes of logics without contraction discussed in the present paper: [13] for intermediate logics (or superintuitionistic logics), [16] for many-valued logics, [30] for fuzzy logics, [25] for residuated lattices, [8, 9] for BCK-algebras and [2I, 25], for substructural logics.

The paper is organized as follows. In Section 2, the logic $F L_{e w}$ and its extesions are introduced. Results on residuated lattices and $\mathrm{FL}_{e w}$-algebras are surveyed in Section 3. Basic results on subdirectly irreducible $\mathrm{FL}_{\mathrm{ew}}$-algebras are shown in Section 4. Some of important extensions of $F L_{e w}$ are introduced in Section 5 as axiomatic systems. In Section 6, simple and semisimple $\mathrm{FL}_{e w^{-}}$ algebras are discussed. A classification of logics over $\mathrm{FL}_{\mathrm{ew}}$ is also introduced 
at the end of the section. Section 7 is devoted to the study of immediate predecessors of the classical logic $\mathrm{Cl}$. It is known that there is a single immediate predecessor of $\mathrm{Cl}$ over the intuitionistic logic, and that there are countably many of them over Łukasiewicz's infinitely many-valued logic. It will be shown there that there are many immediate predecessors of $\mathrm{Cl}$ other than them among logics over $\mathrm{FL}_{\mathrm{ew}}$.

The author owed much to Bob Meyer and Josep M. Font in completing the initial draft of the paper. The author would like to express his special thanks to them for constant encouragement and suggestions. The author is also indebted much to A. Avron, G. Bezhanishvili, W. Blok, M. Bunder, H. Ida, T. Kowalski, K. Swirydowicz and F. Wolter for their advices and helps in various forms.

\section{LOgics Without THE CONTRACTION RULE}

We will introduce first a sequent calculus $F L_{e w}$, which is our basic logic without the contraction rule. Roughly speaking, $\mathrm{FL}_{\mathrm{ew}}$ is the system obtained from Gentzen's sequent calculus LJ for the intuitionistic logic by deleting the contraction rule. The language of $\mathrm{FL}_{\mathrm{ew}}$ consists of a logical constant $\perp$, logical connectives $\rightarrow, \wedge, \vee$ and . (called multiplicative conjunction or fusion). The negation $\neg A$ of a formula $A$ is defined as an abbreviation of $A \rightarrow \perp$. Sometimes, we will abbreviate the formula $(A \rightarrow B) \wedge(B \rightarrow A)$ to $A \equiv B$. A sequent is of the form $A_{1}, \ldots, A_{m} \Rightarrow B$ where $m \geqslant 0$. In the following, capital Greek letters denote finite (possibly empty) sequences of formulas. The system $F L_{e w}$ consists of the following initial sequents

I. $A \Rightarrow A$

2. $\perp, \Gamma \Rightarrow \mathrm{C}$

and the following rules of inference;

Cut rule:

$$
\frac{\Gamma \Rightarrow A \quad A, \Delta \Rightarrow C}{\Gamma, \Delta \Rightarrow C}
$$

Exchange rule and weakening rule:

$$
\frac{\Gamma, A, B, \Delta \Rightarrow C}{\Gamma, B, A, \Delta \Rightarrow C}(\text { ex }) \quad \frac{\Gamma \Rightarrow C}{A, \Gamma \Rightarrow C}(\text { weak })
$$

Rules for logical connectives:

$$
\begin{array}{cc}
\frac{A, \Gamma \Rightarrow B}{\Gamma \Rightarrow A \rightarrow B}(\Rightarrow \rightarrow) & \frac{\Gamma \Rightarrow A \quad B, \Delta \Rightarrow C}{A \rightarrow B, \Gamma, \Delta \Rightarrow C}(\rightarrow \Rightarrow) \\
\frac{\Gamma \Rightarrow A}{\Gamma \Rightarrow A \vee B}(\Rightarrow \vee 1) & \frac{\Gamma \Rightarrow B}{\Gamma \Rightarrow A \vee B}(\Rightarrow \vee 2)
\end{array}
$$




$$
\begin{gathered}
\frac{A, \Gamma \Rightarrow C \quad B, \Gamma \Rightarrow C}{A \vee B, \Gamma \Rightarrow C}(\vee \Rightarrow) \\
\frac{\Gamma \Rightarrow A \quad \Gamma \Rightarrow B}{\Gamma \Rightarrow A \wedge B}(\Rightarrow \wedge) \\
\frac{A, \Gamma \Rightarrow C}{A \wedge B, \Gamma \Rightarrow C}(\wedge 1 \Rightarrow) \quad \frac{B, \Gamma \Rightarrow C}{A \wedge B, \Gamma \Rightarrow C}(\wedge 2 \Rightarrow) \\
\frac{\Gamma \Rightarrow A \quad \Delta \Rightarrow B}{\Gamma, \Delta \Rightarrow A \cdot B}(\Rightarrow \cdot) \quad \frac{A, B, \Gamma \Rightarrow C}{A \cdot B, \Gamma \Rightarrow C}(\cdot \Rightarrow) .
\end{gathered}
$$

The provability of a given sequent is defined in the usual way. In particular, we say that a formula $A$ is provable in $F L_{e w}$ when the sequent $\Rightarrow A$ is provable in it.

In our joint paper [55] with Y. Komori, both syntactic and semantic properties of $F L_{e w}$ are studied. The cut elimination theorem for $F L_{e w}$ is shown, from which both the decidability and Craig's interpolation theorem of $F L_{e w}$ are derived. Also, a Hilbert-style formulation of $F L_{e w}$ is introduced and the separation theorem is proved. Then, a Kripke-type semantics for $F L_{e w}$ and related systems is introduced and their completeness with respect to the semantics is proved in it. (For additional information on $\mathrm{FL}_{\mathrm{ew}}$ and related systems (up to the middle of 9os), see also [22, 23, 52, 53].) It should be also noticed that monoidal propositional logic in [33] discussed in the context of fuzzy logic is equivalent to the logic $\mathrm{FL}_{\mathrm{ew}}$.

Let $\mathrm{FL}_{e}$ be the sequent system obtained from $F L_{e w}$ by deleting the weakening rule, and $F L_{e c}$ be the sequent system obtained from $F L_{e}$ by adding the following contraction rule:

$$
\frac{A, A, \Gamma \Rightarrow C}{A, \Gamma \Rightarrow C}(\text { con }) \text {. }
$$

The system $F L_{e}$ gives a sequent calculus for the intuitionistic linear logic. It is clear that the sequent calculus obtained from $\mathrm{FL}_{\mathrm{ew}}$ by adding the contraction rule gives a (cut-free) sequent calculus for the intuitionistic logic Int. We can see that any sequent of the form $A \cdot B \Rightarrow A \wedge B$ (and of the form $A \wedge B \Rightarrow A \cdot B$ ) is provable in $F L_{e w}$ (and $F L_{e c}$, respectively). Thus in Int, the fusion - becomes equivalent to $\wedge$.

In the present paper, we will concentrate on the study of substructural propositional logics over $\mathrm{FL}_{\mathrm{ew}}$. Here, by a substrctural logic over $\mathrm{FL}_{\mathrm{ew}}$ (or a substructural logic without the contraction rule), we mean any set of formulas which includes all formulas provable in $\mathrm{FL}_{\mathrm{ew}}$ and is closed under substitution and modus ponens, i.e. if both $A$ and $A \rightarrow B$ belong to the set then $B$ belongs also to it. Sometimes, we omit the word "substractual", or even call it simply a $\operatorname{logic} \sqrt{1}$ Also, we identify a formal system with the set of all formulas which are

${ }^{\mathrm{I}}(\dagger)$ As for the definition of substructural logic in general, see $[25]$. 
provable in it, when no confusions will occur. Thus, $\mathrm{FL}_{\mathrm{ew}}$ denotes not only the sequent calculus introduced in the above, but also the set of all formulas provable in it. In the following, $\mathrm{Cl}$ and Int denote classical logic and intuitionistic logic, respectively. Intermediate logics (or, superintuitionistic logics), which are logics over intuitionistic logic, Łukasiewicz's many-valued logics, and fuzzy logics are important examples of logics over $F L_{e w}$, which have been already studied extensively.

The class of logics over $\mathrm{FL}_{\mathrm{ew}}$ is ordered by the set inclusion $\subseteq$. (It is in fact a set, since each logic is a subset of the set of all formulas.) Of course, $F L_{e w}$ is the smallest logic among them, and the set of all formulas is the greatest one, which is the inconsistent logic. We are concerned only with consistent logics, among which the classical logic $\mathrm{Cl}$ is the greatest.

Suppose that $\left\{L_{i}\right\}_{i \in I}$ is a set of logics, where $I$ is a (possibly infinite) nonempty set of indices. Then, clearly the set intersection $\bigcap_{i \in I} L_{i}$ of them is also a logic. Thus, the set of all logics over $\mathrm{FL}_{\mathrm{ew}}$ forms a complete lattice, in which the join $\bigvee_{i \in I} L_{i}$ of $L_{i} s$ is represented as follows.

$$
\begin{aligned}
\bigvee_{i \in \mathrm{I}} \mathrm{L}_{i}= & \left\{\mathrm{A}: \text { there exist } j_{1}, \ldots, j_{k} \in \mathrm{I} \text { and formulas } \mathrm{B}_{j_{\mathrm{t}}} \in \mathrm{L}_{\mathrm{j}_{\mathrm{t}}}\right. \text { for } \\
& 1 \leqslant \mathrm{t} \leqslant \mathrm{k} \text { such that the formula }\left(\mathrm{B}_{j_{1}} \cdot \ldots \cdot \mathrm{B}_{j_{\mathrm{k}}}\right) \rightarrow \mathrm{A} \\
& \text { is provable in } \left.\mathrm{FL}_{\mathrm{ew}}\right\} .
\end{aligned}
$$

THEOREM 2.I The set of all logics over $\mathrm{FL}_{\mathrm{ew}}$ forms a complete lattice, in which the following distributive law hold;

$$
\mathrm{L} \cap \bigvee_{i} \mathrm{~L}_{i}=\bigvee_{i}\left(\mathrm{~L} \cap \mathrm{L}_{i}\right)
$$

The distributivity in the above theorem can be easily shown by using the above representation of joins. We can derive it also from the fact that the variety of $\mathrm{FL}_{\mathrm{ew}}$-algebras is congruence-distributive (see Proposition 3.5), by using the result by Jónsson [39] (see also [5]).

Let $\mathrm{L}_{0}$ and $\mathrm{L}$ be logics such that $\mathrm{L}_{0} \subseteq \mathrm{L}$. Then, $\mathrm{L}$ is said to be finitely axiomatized over $L_{0}$ by the axioms $A_{1}, \ldots, A_{m}$, if $L$ is the smallest logic which contains both $L_{0}$ and the set $\left\{A_{1}, \ldots, A_{m}\right\}$. It holds that for any formula $C$ it is in $L$ if and only if there exist formulas $B_{1}, \ldots, B_{n}$ (for some $n \geqslant 0$ ), each of which is a substitution instance of some $A_{k}$, such that the formula $\left(B_{1} \ldots B_{n}\right) \rightarrow C$ belongs to $L_{0}$. The logic $L$ is denoted by $L_{0}\left[A_{1}, \ldots, A_{m}\right]$ in this case. A logic $L$ is said to be finitely axiomatizable over $L_{0}$ when there exist some axioms by which $L$ is finitely axiomatized over $L_{0}$. We will omit the word "over $L_{0}$ " when $L_{0}$ is $F L_{e w}$. It is easy to see that $L\left[A_{1}, \ldots, A_{m}\right]=L\left[\left(A_{1} \cdot \ldots \cdot A_{m}\right)\right]$, by the help of the weakening rule of $F L_{e w}$, i.e. by using the fact that formulas of the form $(C \cdot D) \rightarrow C$ is always provable in $F L_{e w}$. It is easy to see the following. 
THEOREM 2.2 Suppose that logics $\mathrm{L}$ and $\mathrm{L}^{\prime}$ are finitely axiomatized over $\mathrm{L}_{0}$ by the axioms $\mathrm{A}$ and $\mathrm{B}$, respectively. Then, logics $\mathrm{L} \wedge \mathrm{L}^{\prime}$ and $\mathrm{L} \vee \mathrm{L}^{\prime}$ are finitely axiomatized over $\mathrm{L}_{0}$ by axioms $\mathrm{A} \vee \mathrm{B}$ and $\mathrm{A} \cdot \mathrm{B}$, respectively. (Here, $\mathrm{A}$ and $\mathrm{B}$ are supposed to have no propositional variables in common, by renaming them if necessary.)

\section{Residuated lattices and $\mathrm{FL}_{\text {ew }}$-Algebras}

In this section, we will introduce $\mathrm{FL}_{e w}$-algebras as the algebraic counterparts of

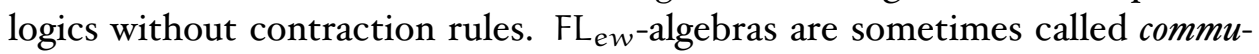
tative integral residuated lattices. Residuated lattices are already introduced and discussed in 3 Os and has been studied by many people, e.g. Krull [47], BalbesDwinger [4], Dilworth [20], Ward-Dilworth [63], Ward [62] and Pavelka [58], $]^{2}$

In our original draft of the present paper in 200I, residuated lattices are defined in a slightly different way. In fact, they should have been called commutative integral residuated lattices, or more precisely $\mathrm{FL}_{e w}$-algebras (see [53]). To avoid confusion, here we start to give a definition of commutative residuated lattices and then give a definition of $\mathrm{FL}_{e w}$-algebras. As for a definition of residuated lattices in general, including noncommutative case, see [25] 3

DEFINITION 3.I An algebra $M=\langle M, \wedge, \vee, \cdot, \rightarrow, 1\rangle$ is a commutative residuated lattice if

- $\langle\mathrm{M}, \wedge, \vee\rangle$ is a lattice,

- $\langle M, \cdot, 1\rangle$ is a commutative monoid with the unit element $\mathrm{I}$,

- for $x, y \in M, x \cdot y \leqslant z$ if and only if $x \leqslant y \rightarrow z$ (the law of residuation).

DEFinition 3.2 An algebra $M=\langle M, \wedge, \vee, \cdot, \rightarrow, 0,1\rangle$ is a $\mathrm{FL}_{e w}$-algebra if

- $\langle M, \wedge, \vee, \cdot, \rightarrow, 1\rangle$ is a commutative residuated lattice,

- $\mathrm{I}$ is the greatest element and $\mathrm{o}$ is the least element.

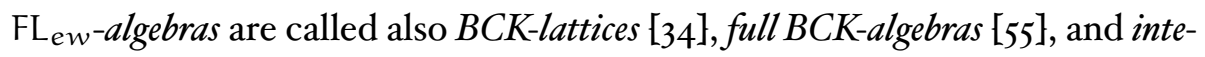
gral, residuated, commutative l-monoids [33]. An extensive study of $\mathrm{FL}_{e w}$-algebras can be seen in [33]. The (dual of the) implicational reduct of an $\mathrm{FL}_{e w}$-algebra is sometimes called a BCK-algebra. A BCK-algebra with the fusion satisfying the law of residuation is called a BCK-algebra with condition (S), in [35]. See also [65], [9] and [10].

\footnotetext{
${ }^{2}$ In $\sqrt[67]{ }$, , Zlatoš discussed residuated lattices and claimed that the class of residuated lattices forms a variety which is arithmetical. Unfortunately, his definition of residuated lattices is insufficient. In fact, from his definition we cannot deduce the law of residuation in Definition 3.2 of residuated lattices. Thus, Proposition I.2 of $[67]$ is incorrect. The present author owes this fact to J. M. Font.

3 By the abuse of symbols, we use the same symbols both for logical connectives and for algebraic operations corresponding to these connectives.
} 
Now we will give a brief survey of basic properties of $\mathrm{FL}_{\mathrm{e} w}$-algebras and of relations between logics over $\mathrm{FL}_{\mathrm{ew}}$ and $\mathrm{FL}_{e w}$-algebras.4 $\mathrm{An}$ the following, we assume that $\mathrm{FL}_{e w}$-algebras are always non-degenerate, i.e. they satisfies $0 \neq 1$. We define $\sim x$ by $\sim x=x \rightarrow 0$. It is easy to see that the following hold in any $\mathrm{FL}_{e w}$-algebra (in fact, the first three hold in any commutative residuated lattice;

(i) $x \leqslant y$ implies $x \cdot z \leqslant y \cdot z$,

(ii) $(x \vee y) \cdot z=(x \cdot z) \vee(y \cdot z)$,

(iii) $1 \rightarrow x=x$,

(iv) $x \cdot y \leqslant x$ and hence $x \cdot y \leqslant x \wedge y$ (integrality),

(v) $x \leqslant y$ if and only if $x \rightarrow y=1$.

Note that the above (ii) expresses the distributivity of · over join, which follows from (i) with the law of residuation. Conversely, suppose that the distributivity (ii) holds in a finite algebra $\mathrm{M}$ satisfying Conditions $\mathrm{I}$ and 2 in the definition of commutative residuated lattices. Then, $M$ becomes a commutative residuated lattice if we define $y \rightarrow z$ by $\max \{x: x \cdot y \leqslant z\}$. In fact, it exists always for all $y, z$, and $\rightarrow$ satisfies the law of residuation.

In the previous section, we mentioned briefly a relationship between logics over $\mathrm{FL}_{\mathrm{ew}}$ and fuzzy logic. We will discuss it here in more detail. In fuzzy set theory, the set of truth values is the unit interval $[0,1]$, which is linearly ordered by the natural order. A binary function $T$ from $[0,1] \times[0,1]$ to $[0,1]$ is called a triangular norm (simply, a t-norm) in the theory of probabilistic metric spaces (see e.g. [60]), if the following holds for $x, y, z$;

I. $\mathrm{T}(x, \mathrm{~T}(\mathrm{y}, z))=\mathrm{T}(\mathrm{T}(x, y), z)$,

2. $T(x, y)=T(y, x)$,

3. $\mathrm{T}(\mathrm{x}, 1)=\mathrm{x}$,

4. $x \leqslant y$ implies $T(x, z) \leqslant T(y, z)$.

Thus, $\langle[0,1], \cdot, 1\rangle$ forms a commutative monoid in which $(i)$ holds, if we define - by $x \cdot y=T(x, y)$. Now we suppose moreover that $T$ is a continuous $t$-norm, i.e. a $t$-norm which is a continuous function over the interval $[0,1]$. For any $y, z$, define a subset $\mathrm{I}_{y, z}$ of $[0,1]$ by $\mathrm{I}_{y, z}=\{x: \mathrm{T}(x, y) \leqslant z\}$ and let $u=\sup \mathrm{I}_{y, z}$. By using the continuity of $T$, we have that $T(u, y)=\sup \left\{T(x, y): x \in I_{y, z}\right\} \leqslant z$. Hence, $u$ is in fact equal to $\operatorname{maxI}_{y, z}$. Thus, the law of residuation holds also, if we define $y \rightarrow z$ by $\operatorname{maxI}_{y, z}$. Therefore, the unit interval with any continuous

${ }^{4}(\dagger)$ By a rapid development of the study in recent years, most of results in this section may be standard now. 
t-norm forms an $\mathrm{FL}_{e w}$-algebra. This gives us a reason why fuzzy logics (in the narrow sense) can be regarded as logics over $\mathrm{FL}_{\mathrm{ew}}$ (see also [30]).5]

Note that in the above only the left-continuity of $T$, i.e. $T\left(\bigvee_{i} x_{i}, y\right)=\bigvee_{i} T\left(x_{i}, y\right)$, is used for showing the existence of residuation $\rightarrow$. It is easily seen that when - is defined by $x \cdot y=T(x, y)$ for all $x, y$ with a leftcontinuous t-norm $T$, the structure $\langle[0,1], \cdot, 1\rangle$ is nothing but an integral, unital commutative quantale with the universe $[0,1]$. (See [59] for more information on quantales. ${ }^{6}$

As we will show later (see Proposition 3.3), the class of all $\mathrm{FL}_{e w}$-algebras determines the logic $\mathrm{FL}_{\mathrm{ew}}$. An $\mathrm{FL}_{e w}$-algebra $\mathrm{M}$ is said to be involutive if

$D N: \sim \sim x=x$ for any $x$

holds always in it. Grišin discussed properties of involutive $\mathrm{FL}_{e w}$-algebras in $[27,28,29]$, where they are called latticed $\mathrm{L}^{\circ}$-algebras (see also [5I], in which they are called Grisin algebras). Also, involutive $\mathrm{FL}_{e w}$-algebras have been studied by E. Casari and P. Minari as algebras for a comparative logic, and they are called Abelian lattice-ordered zeroids. (See a survey of comparative logic in Casari [12].) We can show the following.

LEMMA 3.I In any involutive $\mathrm{FL}_{e w}$-algebra, the following holds.

$$
\begin{aligned}
& \text { I. } \sim(x \vee y)=\sim x \wedge \sim y, \\
& \text { 2. } x \cdot y=\sim(x \rightarrow \sim y) .
\end{aligned}
$$

$\mathrm{FL}_{e}$-algebras are defined similarly to $\mathrm{FL}_{e w}$-algebras, but by deleting the second condition of Definition 3.2, which says that 1 is the greatest element and 0 is the least element. (Thus, 0 is an arbitrary element.) It is well-known that the class of involutive $\mathrm{FL}_{e}$-algebras determines the linear logic (without exponentials) MALL introduced by Girard [26. Complete involutive $\mathrm{FL}_{e}$-algebras (i.e. complete as lattices) are called unital, commutative quantales (see e.g. [59]).

The algebraic condition which corresponds to the contraction rule is $z \leqslant z$. $z$ (square-increasingness). From this, the inequality $x \wedge y \leqslant x \cdot y$ follows. Thus, for each $F L_{e w}$-algebra $M$, the monoid operation - of $M$ is square-increasing, if and only if the monoid operation - of $M$ is equal to $\Lambda$, if and only if $M$ is a Heyting algebra. Another interesting class of algebras related to $\mathrm{FL}_{\mathrm{ew}}$-algebras arose from Łukasiewicz's many-valued logics. Algebras in the class are called by various names, e.g. MV-algebras in [14], Wajsberg algebras in [24], $\mathrm{CN}$-algebras in [4I] and (bounded) commutative BCK-algebras in [66, 36] etc.. (As for the exact relation between MV-algebras and Wajsberg algebras, see e.g. [24].) We will give here a short sketch of them and their connection with $\mathrm{FL}_{e w}$-algebras.

\footnotetext{
${ }^{5}$ See $[2]$ and $\left[\mathrm{II}^{-}\right]$for important results on the logic of continuous t-norms up to $200 \mathrm{I}$.

${ }^{6}(\dagger)$ See also $[25$.
} 
Definition 3.3 An algebra $W=\langle W, \rightarrow, \sim, 1\rangle$ is a Wajsberg algebra if

- $1 \rightarrow x=x$,

- $(x \rightarrow y) \rightarrow((y \rightarrow z) \rightarrow(x \rightarrow z))=1$,

- $(x \rightarrow y) \rightarrow y=(y \rightarrow x) \rightarrow x$,

- $(\sim x \rightarrow \sim y) \rightarrow(y \rightarrow x)=1$.

In a Wajsberg algebra $W$, let us define the binary relation $\leqslant$ on $W$ by $x \leqslant y$ if and only if $x \rightarrow y=1$. Then $\langle W, \leqslant\rangle$ is a partially ordered set with the greatest element $\mathrm{I}$, and the least element 0 if we define 0 by $\sim 1$. Moreover, if we define $x \vee y=(x \rightarrow y) \rightarrow y$ and $x \wedge y=\sim(\sim x \vee \sim y)$ then $x \vee y$ and $x \wedge y$ are equal to the supremum and the infimum of $\{x, y\}$, respectively, with respect to the order $\leqslant$. Hence $W$ forms a lattice. Also, it can be shown that $x \rightarrow 0=\sim x$, $\sim x=x$ and moreover that $(x \rightarrow y) \vee(y \rightarrow x)=1$ (prelinearity). The following equation (I), which is the third condition in Definition 3.3. plays an important role as shown below.

$$
(x \rightarrow y) \rightarrow y=(y \rightarrow x) \rightarrow x .
$$

It is easy to see that any $\mathrm{FL}_{e w}$-algebra satisfying (I) is involutive. (As for the details of the above discussions, see e.g. [24]. See also $[8]$ for related topics.) The following proposition is proved in [24] and [49].

Proposition 3.I Let $\mathrm{W}=\langle\mathrm{W}, \rightarrow, \sim, 1\rangle$ be a Wajsberg algebra. Define a binary relation $\leqslant$ on $\mathrm{W}$ as above, and a binary operation by $\mathrm{x} \cdot \mathrm{y}=\sim(\mathrm{x} \rightarrow \sim \mathrm{y})$. Then, $\mathrm{W}=\langle\mathrm{W}, \wedge, \vee, \cdot, \rightarrow, 0,1\rangle$ forms a (involutive) $\mathrm{FL}_{\mathrm{ew}}$-algebra. Conversely, each $\mathrm{FL}_{\mathrm{ew}} \mathrm{w}^{-}$ algebra satisfying $(\mathrm{x} \rightarrow \mathrm{y}) \rightarrow \mathrm{y}=(\mathrm{y} \rightarrow \mathrm{x}) \rightarrow \mathrm{x}$ naturally determines a Wajsberg algebra.

The above proposition assures us that Wajsberg algebras can be regarded as $\mathrm{FL}_{e w}$-algebras. Wajsberg algebras are originally introduced as models of Łukasiewicz's many-valued logics. Let us define the following operations either on the set $\{0,1 / n, 2 / n, \ldots,(n-1) / n, 1\}$ for a positive integer $n$ or on the unit interval $[0,1]$.

$$
\begin{aligned}
& \text { - } x \rightarrow y=\min \{1,1-x+y\}, \\
& \text { - } \sim x=x \rightarrow 0=1-x, \\
& \text { - } x \cdot y=\sim(x \rightarrow \sim y)=\max \{0, x+y-1\} .
\end{aligned}
$$

Then in either case, it forms a Wajsberg algebra. They are denoted as $\succeq_{n+1}$ and $€$, respectively, in the present paper. We have also the following (see also Lemmas 2.I4 and 2.5 of [33]).

PROPOSITION 3.2 For any $\mathrm{FL}_{e w}$-algebra $\mathrm{M}, \mathrm{M}$ satisfies the equation (I) if and only if it is involutive and satisfies $\mathrm{x} \wedge \mathrm{y}=\mathrm{x} \cdot(\mathrm{x} \rightarrow \mathrm{y})$. 
Proof: The only-if part can be obtained by using equations in Lemma 3.I The if-part holds, since $x \vee y=\sim(\sim x \wedge \sim y)=(\sim x \cdot(\sim x \rightarrow \sim y)) \rightarrow 0=((y \rightarrow x) \cdot \sim x$ )$\rightarrow 0=(y \rightarrow x) \rightarrow(\sim x \rightarrow 0)=(y \rightarrow x) \rightarrow x$. Similarly, $x \vee y=y \vee x=(x \rightarrow$ $y) \rightarrow y$. Thus, equation (I) holds.

Any $\mathrm{FL}_{\mathrm{e} w}$-algebra satisfying the equation $(x \rightarrow y) \vee(y \rightarrow x)=1$ is called an MLT-algebra. An MLT-algebra satisfying the equation $x \wedge y=x \cdot(x \rightarrow$ $y$ ) is called a BL-algebra (see [30]). By Proposition 3.2. Wajberg algebras are (essentially) equal to involutive BL-algebras.

In the usual way, we will define the validity of formulas (of $F L_{e w}$ ) in a given $\mathrm{FL}_{e w}$-algebra $\mathrm{M}$ as follows. Any mapping $v$ from the set of all propositional variables to the set $M$ is called a valuation on $M$. A given valuation $v$ can be extended to a mapping from the set of all formulas to $M$, inductively as follows.

I. $v(\perp)=0$,

2. $v(A \wedge B)=v(A) \wedge v(B)$,

3. $v(\mathrm{~A} \vee \mathrm{B})=v(\mathrm{~A}) \vee v(\mathrm{~B})$,

4. $v(\mathrm{~A} \cdot \mathrm{B})=v(\mathrm{~A}) \cdot v(\mathrm{~B})$

5. $v(\mathrm{~A} \rightarrow \mathrm{B})=v(\mathrm{~A}) \rightarrow v(\mathrm{~B})$.

A formula $A$ is valid in $M$ if $v(A)=1$ holds for any valuation on $M$. The set of formulas which are valid in $M$ is denoted by $L(M)$. Next, a given sequent $A_{1}, \ldots, A_{m} \Rightarrow B$ is said to be valid in $M$ if the formula $\left(A_{1} \cdot \ldots \cdot A_{m}\right) \rightarrow B$ is valid in it. Then, the following completeness theorem of $F L_{e w}$ can be shown in a standard way.

Proposition $3.3 A$ sequent $S$ is provable in $\mathrm{FL}_{\mathrm{ew}}$ if and only if it is valid in all $\mathrm{FL}_{\text {ew-algebras. }}$

It is easy to see that $L(M)$ is a logic over $F L_{e w}$ for any $F L_{e w}$-algebra $M$, which is called the logic determined by M. (In general, for any class $\mathcal{K}$ of $F L_{e w}$-algebras, the logic $\bigcap_{M \in \mathcal{K}} L(M)$ is a logic over $F L_{\text {ew }}$, called the logic determined by $\mathcal{K}$.) Conversely, for any logic $\mathrm{L}$ over $\mathrm{FL}_{\mathrm{ew}}$ there exists an $\mathrm{FL}_{e w}$-algebra $\mathrm{M}$ such that $\mathrm{L}=\mathrm{L}(\mathrm{M})$. In fact, it is enough to take the Lindenbaum algebra of $\mathrm{L}$ for $\mathrm{M}$.

Blok and Ferreirim have developed the study of algebras called hoops, in [6, 77. Each hoop satisfies the equation $x \cdot(x \rightarrow y)=y \cdot(y \rightarrow x)$. When a hoop satisfies moreover the equation (I), it is called a Wajsberg hoop. Any $\mathrm{FL}_{e w^{-}}$ algebra with the equation (I) is an example of a Wajsberg hoop. As for the details, see [6, 77.

In [64], Wroński proved that the class of all BCK-algbras does not form a variety] (See also Higgs [32].) On the other hand, Idziak [34] proved the following.

${ }^{7}(\dagger)$ For basic notoins and results on universal algebra, see [I]. 
PROPOSITION 3.4 The class of all $\mathrm{FL}_{\mathrm{ew}}$-algebras forms a variety.

A variety $\mathcal{K}$ is congruence-distributive when the lattice of all congruence relations of any algebra in $\mathcal{K}$ is distributive, and is congruence permutable when every two congruences of any algebra in $\mathcal{K}$ permute. Moreover, if $\mathcal{K}$ is both congruence-distributive and congruence permutable, it is said to be arithmetical. Then the following holds (see [34]).

PROPOSITION 3.5 The variety of $\mathrm{FL}_{\mathrm{ew}}$-algebras is arithmetical.

Next, we will give the definition of filters of $\mathrm{FL}_{e w}$-algebras 8

DEFINITION 3.4 A nonempty subset $\mathrm{F}$ of an $\mathrm{FL}_{e w}$-algebra $\mathrm{M}$ is an implicative filter (or, simply a filter) if for $x, y \in M$ it satisfies

- $x \leqslant y$ and $x \in$ F imply $y \in F$,

- $x, y \in F$ implies $x \cdot y \in F$.

Note that any filter $F$ of an $F L_{e w}$-algebra satisfies that $x, y \in F$ implies $x \wedge y \in F$. It is easy to see that a nonempty subset $F$ of a residuated lattice is a filter if and only if it satisfies that $\mathrm{I}) \quad 1 \in \mathrm{F}$ and 2) $x, x \rightarrow y \in F$ implies $y \in F$ (see e.g. [24]). Let $S$ be a nonempty subset of an $F L_{e w}$-algebra $M$. Then the set $\left\{x: a_{1} \cdots a_{k} \leqslant x\right.$ for some $\left.a_{1}, \ldots, a_{k} \in S\right\}$ is shown to be a filter, called the filter generated by $S$. In particular, the filter generated by a singleton set $\{a\}$ for an element $a \in M$ will be expressed as $\left\{x: a^{k} \leqslant x\right.$ for some positive integer $k\}$, where $a^{k}$ denotes $a \cdots a$ with $k$ times $a$. The following can be shown easily (see e.g. [33].).

PROPOSITION 3.6 Let $\mathrm{M}$ be any $\mathrm{FL}_{\mathrm{ew}}$-algebra. Then, there exists a lattice isomorphism between the set of all filters of $\mathrm{M}$ and the set of all congruences of $\mathrm{M}$.

In fact, for a given filter $F$ the binary relation $\sim_{F}$ defined by

$x \sim F y$ if and only if $x \rightarrow y, y \rightarrow x \in F$

is a congruence and the map $\theta$ defined by $\theta(F)=\sim_{F}$ is the required map. The converse map is defined by taking the filter $\{x: x \sim 1\}$ for a given congruence $\sim$.

\section{Subdirectly irreducible FL $_{e w}$-Algebras}

Let $M$ and $N_{i}$ for each $i \in I$ be residuated lattices. By a subdirect representation of $M$ with factors $N_{i}$ we mean an embedding $f$ from $M$ to the product $\prod_{i \in I} N_{i}$ such that each $f_{i}$ defined by $f_{i}=p_{i} \circ f$ is onto $N_{i}$ for each $i \in I$. Here, $p_{i}$ denotes the $i$-th projection. An $\mathrm{FL}_{\mathrm{ew}}$-algebra $\mathrm{M}$ is subdirectly irreducible if it is non-degenerate and for any subdirect representation $f: M \rightarrow \prod_{i \in I} N_{i}$, there exists a $j$ such that $f_{j}$ is an isomorphism of $M$ onto $N_{j}$ (see [48) for the details).

\footnotetext{
${ }^{8}(\dagger)$ In $[25]$, they are called deductive filters. For the definition of filters in arbitrary residuated lattices, see $[25$. Filter generation and its relation to (generalized form of) deduction theorem are also discussed in it.
} 
From Birkhoff's subdirect representation theorem it follows that every $\mathrm{FL}_{e w}$-algebra has a subdirect representation with subdirectly irreducible $\mathrm{FL}_{e w^{-}}$ algebras. By using Proposition 3.6, we can see that an $\mathrm{FL}_{e w}$-algebra $\mathrm{M}$ is subdirectly irreducible if and only if it has the second smallest filter, i.e. the smallest filter among all filters except $\{1\}$. Note that when an $F L_{e w}$-algebra $M$ is subdirectly represented by the set $\left\{\mathrm{N}_{j}\right\}_{j \in J}$ of $F L_{e w}$-algebras, the logic $L(M)$ determined by $M$ can be expressed as $\bigcap_{j \in J} L\left(N_{j}\right)$.

We can easily show the following lemma which gives a necessary and sufficient condition for an $\mathrm{FL}_{\mathrm{ew}}$-algebra to be subdirectly irreducible.

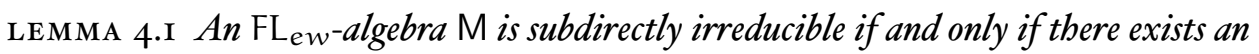
element a $(<1)$ such that for any $x<1$ there exists a positive integer $m$ for which $\mathrm{x}^{\mathrm{m}} \leqslant$ a holds.

Using this lemma, we can show the following.

THEOREM 4.I In any subdirectly irreducible $\mathrm{FL}_{\mathrm{e} w}$-algebra, if $\mathrm{x} \vee \mathrm{y}=1$ then either $\mathrm{x}=1$ or $\mathrm{y}=1$ bolds.

Proof: By taking the contraposition, it suffices to show that $x, y<1$ implies $x V$ $y<1$ in a given subdirectly irreducible $F L_{e w}$-algebra $M$. Since $M$ is subdirectly irreducible, there exists $a<1$ such that for any $z<1$ there exists a number $k$ satisfying $z^{k} \leqslant a$. In particular, both $x^{m} \leqslant a$ and $y^{n} \leqslant a$ hold for some positive integers $m$ and $n$. Define $s=\max \{m, n\}$ and $t=2 s-1$. Then, clearly $x^{s} \leqslant a$ and $y^{s} \leqslant a$ hold. Now, by the distributivity of $\cdot$ with $\vee$,

$$
(x \vee y)^{t}=\bigvee_{i=1}^{t} x^{i} \cdot y^{t-i}
$$

It is easy to see that either $i \geqslant s$ or $t-i \geqslant s$. Hence, in the former case,

$$
x^{i} \cdot y^{t-i} \leqslant x^{i} \leqslant x^{s} \leqslant a
$$

and in the latter case,

$$
x^{i} \cdot y^{t-i} \leqslant y^{t-i} \leqslant y^{s} \leqslant a .
$$

Thus, in either case, $(x \vee y)^{t} \leqslant a$. Therefore, $x \vee y$ cannot be equal to 1 .

An element $a$ in an $\mathrm{FL}_{e w}$-algebra $\mathrm{M}$ is a coatom if it is maximal among elements in $M-\{1\}$. Then we have the following immediately from the above theorem.

COROLLARY 4.I Every subdirectly irreducible $\mathrm{FL}_{e}$-algebra bas either the single coatom or no coatoms.

The following result is essentially due to Kowalski $[42]$, which makes an interesting contrast with Lemma 4.I.

LEMMA 4.2 An $\mathrm{FL}_{e w}$-algebra $\mathrm{M}$ bas the unique coatom if and only if there exists an element $\mathrm{a}(<1)$ and a positive integer $\mathrm{m}$ such that $\mathrm{x}^{\mathrm{m}} \leqslant \mathrm{a}$ bolds for any $\mathrm{x}<1$. 
Proof: To show the only-if part, it is enough to take the coatom for a and I for $m$. Conversely, suppose that there exists $a(<1)$ and $m \geqslant 1$ such that $x^{m} \leqslant a$ for any $x<1$. By Lemma 4.I. it is clear that $M$ is subdirectly irreducible. Now, take any such $a$ and take also the smallest number $k$ among such ms for this $a$. If $k=1$ then it is obvious that $a$ is the single coatom of M. So, suppose that $k>1$. By our assumption, there exists an element $b$ such that $b^{k-1} \leqslant a$ but $b^{k} \leqslant a$. Define $d$ by $d=b^{k-1} \rightarrow a$. Clearly, $d<1$. We will show that $d$ is the single coatom of $M$. Take any $y$ such that $y<1$ and let $z=d \vee y$. Then, $d, y \leqslant z$ and moreover $z<1$ by Theorem 4.I Since $b^{k} \leqslant a$, $\mathrm{b} \leqslant \mathrm{b}^{\mathrm{k}-1} \rightarrow \mathrm{a}=\mathrm{d} \leqslant z$. As $z<1$, we have $z^{\mathrm{k}} \leqslant \mathrm{a}$ by our assumption. Thus, $y \leqslant z \leqslant z^{k-1} \rightarrow a \leqslant b^{k-1} \rightarrow a=d$. Therefore, $y \leqslant d$. Hence, $d$ is the coatom of $\mathrm{M}$.

Let us consider the following condition on $\mathrm{FL}_{e w}$-algebras for a given positive integer $k$ :

$$
E_{\mathrm{k}}: x^{\mathrm{k}+1}=x^{\mathrm{k}} \text { for any } \mathrm{x} .
$$

The condition $E_{k}$ is equivalent to the condition that $x^{k+1} \rightarrow y=x^{k} \rightarrow y$ for all $x, y$ (see e.g. [7] ]). It is introduced and discussed by Cornish [I8]. Sometimes, $\mathrm{M}$ is said to be k-potent when it satisfies $E_{\mathrm{k}}$. It is obvious that every finite $\mathrm{FL}_{\mathrm{ew}}$-algebra is k-potent for some $\mathrm{k}$.

COROLLARY 4.2 If a subdirectly irreducible $\mathrm{FL}_{e w}$-algebra $\mathrm{M}$ satisfies $\mathrm{E}_{\mathrm{k}}$ for some $\mathrm{k}$, then it has the unique coatom.

Proof: Suppose that $M$ satisfies $E_{\mathrm{k}}$. Since $M$ is subdirectly irreducible, there exists an element $a(<1)$ such that for each $x<1$ there exists a positive integer $m$ such that $x^{m} \leqslant a$ holds. Then $x^{k} \leqslant a$ must hold also for each $x<1$. Thus, by Lemma $4.2 \mathrm{M}$ has the unique coatom.

A related result for BCK-algebras was shown in [57]. From the above lemma, it follows immediately that every finite subdirectly irreducible $\mathrm{FL}_{\mathrm{ew}}{ }^{-}$ algebra has a single coatom. It is easy to see that a subdirectly irreducible $\mathrm{FL}_{e w}$-algebras with the unique coatom does not always satisfy $E_{\mathrm{k}}$ for some k. In the following, we will give an interesting example of a subdirectly irreducible $\mathrm{FL}_{e w}$-algebra with no coatoms. This special $\mathrm{FL}_{e w}$-algebra has been discussed by Pavelka [58], Hájek, Godo and Esteva [3I] etc. in connection with fuzzy logic.

Let $M$ be the interval $[0,1]$, i.e. the set of all real numbers between 0 and 1 . Then $M$ forms a bounded lattice with the natural order $\leqslant$. Moreover, $\langle M, \times, 1\rangle$ is a commutative monoid satisfying $x \times y \leqslant x$, where $x$ denotes the usual multiplication. Since $M$ is linearly ordered, the distributivity of $\times$ with $\vee$ follows from the above inequality. Now, define a binary operation $\rightarrow$ on $M$ by

$$
y \rightarrow z= \begin{cases}z / y & \text { if } y>z \\ 1 & \text { otherwise }\end{cases}
$$


Then, we can show that $x \times y \leqslant z$ if and only if $x \leqslant y \rightarrow z$. Thus, $M$ forms an $\mathrm{FL}_{e w}$-algebra. Clearly, $M$ has no coatoms. Now, let a be an arbitrary real number such that $0<a<1$. Then, we can show that for each $r<1$ there exists a positive number $m$ such that $r^{m} \leqslant a$. This means that $M$ is subdirectly irreducible.

The logic determined by the above $\mathrm{FL}_{e w}$-algebra is called product logic, whose axiomatization is given in [3I]. A class of $\mathrm{FL}_{e w}$-algebras related to product logic, called product algebras, is discussed in [I, 30, 3I] etc. 9

The following fact, remarked in [3], should be pointed out here. Suppose that $a$ is a real number such that $0<a<1$. define an operation $\circ$ on the interval $[a, 1]$ by

$$
x \circ y=\max \{a, x \times y\} .
$$

Then, this with the operation $\rightarrow$ satisfying

$$
y \rightarrow z=z / y \text { if } y>z \text {, and }=1 \text { otherwise. }
$$

determines an $\mathrm{FL}_{e w}$-algebra, which we call $\mathrm{I}_{\mathrm{a}}$. Obviously, this is also a subdirectly irreducible $\mathrm{FL}_{e w}$-algebra without coatoms. The logic determined by this $\mathrm{FL}_{e w}$-algebra is equal to Łukasiewicz's infinitely many-valued logic. In fact, the mapping $\phi$ from $[0,1]$ to $[a, 1]$ defined by $\phi(x)=a^{1-x}$ is a monotone increasing map satisfying $\phi(0)=a, \phi(1)=1$ and $\phi(\max \{0, x+y-1\})=\phi(x) \circ \phi(y)$, and hence it is an isomorphism (see also [30]).

In the dual form, this fact can be restated as follows. Let $\mathrm{R}$ be the set of all real numbers as an additive ordered Abelian group. Then the mapping $\psi$ from the MV-algebra R[I] (see Chang [I5] for the definition) to $l_{a}$ by $\psi(x)=a^{x}$ is an isomorphism.

Let Lin be the formula $(p \rightarrow q) \vee(q \rightarrow p)$, which is sometimes called the axiom of prelinearity. It is called also the (algebraic) strong de Morgan law in $[38$. and [59]. Using Theorem 4.I we can show the following (see also [33] Theorem 4.8).

LEMMA 4.3 For any subdirectly irreducible $\mathrm{FL}_{\text {ew }}$-algebra $\mathrm{M}$, the formula Lin is valid in $\mathrm{M}$ if and only if $\mathrm{M}$ is linearly ordered.

Proof: The if-part is trivial. Suppose that Lin is valid in M. This implies that $(a \rightarrow b) \vee(b \rightarrow a)=1$ for all $a, b \in M$. By Theorem 4.I. either $a \rightarrow b=1$ or $b \rightarrow a=1$ holds. Thus, either $a \leqslant b$ or $b \leqslant a$. Hence, $M$ is linearly ordered.

COROLLARY 4.3 If the formula Lin is valid in an $\mathrm{FL}_{e}$-algebra $\mathrm{M}$ then $\mathrm{M}$ is a distributive lattice.

Proof: Suppose that $\mathrm{M}$ is subdirectly represented by the set of subdirectly irreducible $F L_{e w}$-algebras $N_{j}$ for $j \in J$. Then, Lin is valid in any $N_{j}$. Therefore,

${ }^{9}(\dagger)$ There are considerable developments in the study of product logic after $200 \mathrm{I}$. 
by Lemma 4.3 each $N_{j}$ is linearly ordered and hence is distributive. In other words, the formula Dis : $(p \wedge(q \vee r)) \rightarrow(p \wedge q) \vee(p \wedge r)$ is valid in each of them. Thus, Dis is valid in $M$ and hence $M$ is distributive.

As for a direct proof of the above corollary, see Corollary I of Proposition 4.3.4 in [59], or Lemma 2.4 (3) in [33].

Suppose that $\mathrm{M}$ and $\mathrm{N}$ are finite subdirectly irreducible $\mathrm{FL}_{e w}$-algebras. It is interesting to see when they determine the same logics. For this purpose, we will show a theorem in the following which is obtained by modifying the proof of Jankov's theorem for Heyting algebras (see [37]).

Let $\mathrm{M}$ be an arbitrary finite, subdirectly irreducible $\mathrm{FL}_{e w}$-algebra and $\omega$ be the coatom of $M$. For each element $x \in M$, take a propositional variable $p_{x}$ in such a way that $p_{y}$ and $p_{z}$ are distinct whenever $y \neq z$. Define a set of formulas $\Delta_{\mathrm{M}}$ by

$$
\begin{aligned}
\Delta_{M}= & \left\{\left(p_{x} \wedge p_{y}\right) \equiv p_{x \wedge y},\left(p_{x} \vee p_{y}\right) \equiv p_{x \vee y},\left(p_{x} \cdot p_{y}\right) \equiv p_{x \cdot y}\right. \\
& \left.\left(p_{x} \rightarrow p_{y}\right) \equiv p_{x \rightarrow y},\left(\neg p_{x}\right) \equiv p_{\sim x}: x, y \in M\right\}
\end{aligned}
$$

Let $\left(\Delta_{M}\right)^{\dagger}$ be the conjunction of all formulas in $\Delta_{M}$. For each positive integer $k$, define the formula $X_{M}(k)$, called the fankov formula of order $k$ for $M$, by $X_{M}(k)=$ $\left(\left(\Delta_{M}\right)^{\dagger}\right)^{k} \rightarrow p_{\omega}$ IO Then, we have the following Jankov's theorem for $\mathrm{FL}_{e w^{-}}$ algebras.

THEOREM 4.2 Let $\mathrm{M}$ be a finite, subdirectly irreducible $\mathrm{FL}_{e w}$-algebra, and let $\mathrm{N}$ be any $\mathrm{FL}_{\text {ew }}$-algebra satisfying $\mathrm{E}_{\mathrm{k}}$. Then, $\mathrm{X}_{\mathrm{M}}(\mathrm{k})$ is not valid in $\mathrm{N}$ if and only if $\mathrm{M}$ is embeddable into a quotient algebra of $\mathrm{N}$.

Proof: Suppose that $h$ is an embedding of $M$ into a quotient algebra N/F of N. Define a valuation $v$ on N/F by $v\left(p_{x}\right)=h(x)$ for each $x \in M$. Then, it is clear that $v\left(X_{M}(k)\right)=1 \rightarrow h(\omega)=h(\omega)<1$. Thus, $X_{M}(k)$ is not valid in N/F, and nor is it in N. Conversely, suppose that the formula $X_{M}(k)$ becomes false under a valuation $w$ on $\mathrm{N}$. Let $w\left(\left(\Delta_{\mathrm{M}}\right)^{\dagger}\right)=\mathrm{d}$ and $w\left(p_{\omega}\right)=e$. By our assumption, $\mathrm{d}^{\mathrm{k}} \leqslant$ e. Since $N$ satisfies $E_{\mathrm{k}}$, the set $\mathrm{G}$ defined by $\mathrm{G}=\left\{x: \mathrm{d}^{\mathrm{k}} \leqslant x\right\}$ forms a filter such that $e \notin \mathrm{G}$. Now, consider the quotient algebra $N / G$ of $N$ and its valuation $w^{*}$ defined by $w^{*}(\mathrm{q})=\|w(\mathrm{q})\|$ for every propositional variable q. Here $\|u\|$ denotes the equivalence class induced by $G$, to which $u$ belongs. Then $w^{*}\left(\left(\Delta_{\mathrm{M}}\right)^{\dagger}\right)=1$ and $w^{*}\left(p_{\omega}\right)=\|e\|<1$. From this, it follows that the mapping $g: M \rightarrow N / G$, defined by $g(x)=w^{*}\left(p_{x}\right)$ for each $x \in M$, is an embedding.

By assuming moreover that $\mathrm{M}$ satisfies also $E_{k}$, we can give another condition which is equivalent to either of the conditions in the above theorem.

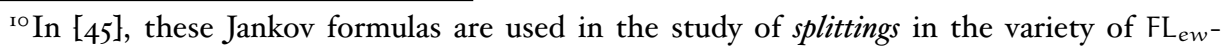
algebras.
} 
COROLlary 4.4 Suppose that $\mathrm{M}$ is a finite, subdirectly irreducible $\mathrm{FL}_{\mathrm{ew}}$-algebra and $\mathrm{N}$ is an arbitrary $\mathrm{FL}_{\mathrm{ew}}$-algebra. When both satisfy $\mathrm{E}_{\mathrm{k}}, \mathrm{M}$ is embeddable into a quotient algebra of $\mathrm{N}$ if and only if $\mathrm{L}(\mathrm{N}) \subseteq \mathrm{L}(\mathrm{M})$.

Proof: The only-if part is trivial. Suppose that $L(N) \subseteq L(M)$. Since $M$ satisfies $E_{\mathrm{k}}, X_{\mathrm{M}}(\mathrm{k})$ is not valid in $\mathrm{M}$. Hence it is not valid in N, either. Then, by Theorem 4.2 , there is an embedding of $\mathrm{M}$ into a quotient algebra of $\mathrm{N}$.

A Heyting algebra is an $\mathrm{FL}_{e w}$-algebra satisfying $E_{1}$, i.e. $x^{2}=x$. Theorem 4.2 with Corollary 4.4 gives us the original form of Jankov's theorem for Heyting algebras.

Let $E_{k}$ be the formula $p^{k} \rightarrow p^{k+1}$ for any $k>0$, where $A^{j}$ denotes the formula $A \cdot \ldots$. A with $j$ times $A$. Obviously, the formula $E_{k}$ is a syntactic expression of $E_{\mathrm{k}}$.

COROLla Ry 4.5 Suppose that both $\mathrm{M}$ and $\mathrm{N}$ are finite subdirectly irreducible $\mathrm{FL}_{\mathrm{ew}}{ }^{-}$ algebras. If the logic $\mathrm{L}(\mathrm{M})$ is equal to $\mathrm{L}(\mathrm{N})$ then $\mathrm{M}$ is isomorphic to $\mathrm{N}$.

Proof: Suppose that $L(M)$ is equal to $L(N)$. Since $M$ is finite, we can assume that $\mathrm{E}_{\mathrm{k}}$ is in $\mathrm{L}(\mathrm{M})$ (and hence in $\mathrm{L}(\mathrm{N})$ ) for some $\mathrm{k}$. By Corollary 4.4, there is an embedding $h$ of $M$ into a quotient algebra of $N$. Hence, $|M| \leqslant|N|$, where $|S|$ denotes the cardinality of a set $S$, and $M$ and $N$ are universes of algebras $M$ and $\mathrm{N}$, respectively. Similarly, since there is an embedding of $\mathrm{N}$ into a quotient algebra of $M,|N| \leqslant|M|$, and hence $|M|=|N|$. Thus $h$ must be an isomorphism of $\mathrm{M}$ onto $\mathrm{N}$.

\section{Some axiomatizable extensions of $\mathrm{FL}_{\mathrm{ew}}$}

To give an overview of the structure of all logics over $F L_{e w}$, we will introduce some basic logics among them, which are finitely axiomatizable. Let us consider the following axioms:

EM: $p \vee \neg p \quad$ (excluded middle)

$\mathrm{DN}: \neg \neg p \rightarrow p \quad$ (double negation)

Con: $(p \rightarrow(p \rightarrow q)) \rightarrow(p \rightarrow q) \quad$ (contraction)

WCon: $(p \rightarrow \neg p) \rightarrow \neg p \quad$ (weak contraction)

$P: \quad((p \rightarrow q) \rightarrow p) \rightarrow p \quad$ (Peirce's law)

WP: $(\neg p \rightarrow p) \rightarrow p \quad$ (weak Peirce's law)

Lin: $(p \rightarrow q) \vee(q \rightarrow p) \quad$ (prelinearity)

Dis: $(p \wedge(q \vee r)) \rightarrow((p \wedge q) \vee(p \wedge r)) \quad$ (distributive law) 
Note that WCon and WP are obtained from Con and P, respectively, by replacing $q$ by $\perp$. The axiom Con will play essentially the same role as the contraction rule (and also as the formula $\mathrm{E}_{1}$ introduced in the previous section). Therefore $\mathrm{FL}_{\mathrm{ew}}[\mathrm{Con}]$ is equal to the intuitionistic logic Int, which is equal also to $F \mathrm{~L}_{\mathrm{ew}}$ with the axiom $p \rightarrow p^{2}$. On the other hand, WCon is equivalent to $\neg p^{2} \rightarrow \neg p$, which is the contrapositive of $p \rightarrow p^{2}$, and $F L_{e w}$ [WCon] is equal to $F L_{e w}$ with the axiom $\neg(p \wedge \neg p)$. It is clear that $F L_{e w}[D N]$ is equal to the logic without the contraction rule studied by Grišin.

LEMMA 5.I Each of the logics $\mathrm{FL}_{\mathrm{ew}}[W P], \mathrm{FL}_{\mathrm{ew}}[P], \mathrm{FL}_{\mathrm{ew}}[E M]$ and $\mathrm{FL}_{\mathrm{ew}}[$ WCon, DN] is equal to classical logic $\mathrm{Cl}$.

Proof: As mentioned above, any instance of WP is provable in $\mathrm{FL}_{\mathrm{ew}}[\mathrm{P}]$. It is obvious that any instance of $\mathrm{P}$ is provable in $\mathrm{Cl}$. To show that each formula $\mathrm{A}$ provable in $\mathrm{Cl}$ is also provable in $\mathrm{FL}_{\mathrm{ew}}[\mathrm{EM}]$, it is enough to show that Con is provable in $\mathrm{FL}_{e w}[\mathrm{EM}]$, since $\mathrm{FL}_{\mathrm{ew}}[\mathrm{Con}]$ is equal to Int and $\operatorname{lnt}[\mathrm{EM}]$ is equal to $\mathrm{Cl}$. We note that the formula $(\neg \mathrm{r} \vee s) \equiv(r \rightarrow s)$ is provable in $\mathrm{FL}_{e w}[\mathrm{EM}]$, using the fact that

$$
(\mathrm{r} \vee \neg \mathrm{r}) \rightarrow((\mathrm{r} \rightarrow \mathrm{s}) \rightarrow(\neg \mathrm{r} \vee \mathrm{s}))
$$

is provable in $F L_{e w}$. Thus,

$$
((p \rightarrow(p \rightarrow q))) \rightarrow(p \rightarrow q)) \equiv((\neg p \vee \neg p \vee q) \rightarrow(\neg p \vee q))
$$

is provable. But, the righthand side of the above equivalence is always provable. Hence, Con is provable in $\mathrm{FL}_{e w}[\mathrm{EM}]$. To show that $\mathrm{EM}$ is provable in $\mathrm{FL}_{\text {ew }}[\mathrm{WP}]$, let $\mathrm{Q}$ be $p \vee \neg p$. Now, since $\neg \mathrm{Q} \rightarrow \mathrm{Q}$ is provable in $F L_{\text {ew }}$ and $(\neg \mathrm{Q} \rightarrow \mathrm{Q}) \rightarrow \mathrm{Q}$ is an instance of WP, we have that $\mathrm{Q}$, i.e. $p \vee \neg p$, is provable in $\mathrm{FL}_{\mathrm{ew}}[\mathrm{WP}]$.

Lastly, we will show that $\mathrm{FL}_{e w}$ [WCon,DN] is equal to $\mathrm{Cl}$. Since $\mathrm{FL}_{e w}[\mathrm{Con}]$ is equal to Int, it is enough to show that $p \rightarrow p^{2}$ is provable in $F L_{e w}$ [WCon,DN]. By WCon, $\neg p^{2} \rightarrow \neg p$ is provable. By taking the contraposition and using DN, $p \rightarrow p^{2}$ follows. This completes our proof.

Note that $\mathrm{FL}_{e}[\mathrm{P}]$ is in fact equal to $\mathrm{Cl}$ as the weakening rule is derivable in it. (This is due to K. Fujita.) A part of the above lemma is essentially obtained in Theorem 7.3 I of [63], which says that complemented $\mathrm{FL}_{\mathrm{ew}}$-algebras are exactly same as Boolean algebras. It can be shown (by using e.g. the cut elimination theorem) that Grišin's logic $F L_{e w}[D N]$ is strictly weaker than the classical logic, and hence $\mathrm{EM}$ is not provable in $\mathrm{FL}_{\mathrm{ew}}[\mathrm{DN}]$. Also, we can show that $\mathrm{EM}$ is provable in $\mathrm{FL}_{\mathrm{e}}$ [WCon,DN]. (On the other hand, probably $\mathrm{DN}$ will not be provable in $\mathrm{FL}_{\mathrm{ec}}[\mathrm{EM}]$.)

Figure 5.I shows the inclusion relationship between logics mentioned in the above. In the following, we will show that all of the inclusions described in the figure are proper. 


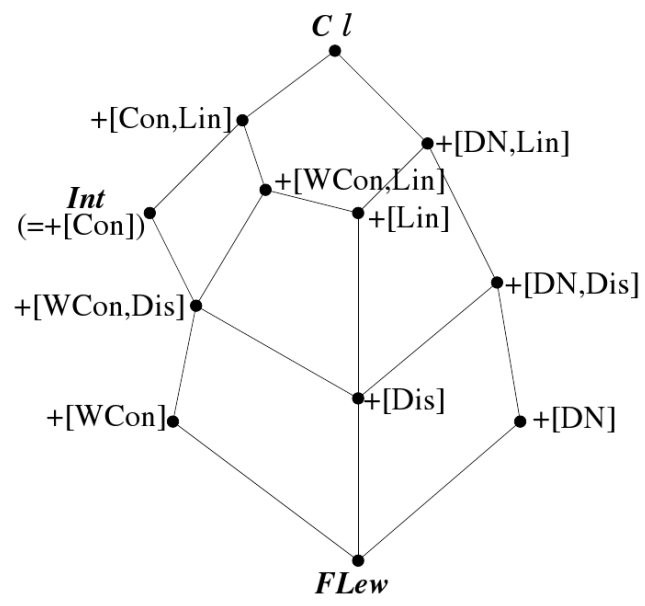

Figure 5.I.

(I) The logic $\mathrm{FL}_{\mathrm{ew}}[\mathrm{Con}, \mathrm{Lin}]$, i.e. Int [Lin], is Gödel logic, which is strictly weaker than $\mathrm{Cl}$. Thus, $\mathrm{FL}_{e w}[\operatorname{Lin}], \mathrm{FL}_{\mathrm{ew}}[\mathrm{Dis}]$, and $\mathrm{FL}_{\mathrm{ew}}$ are strictly weaker than $\mathrm{FL}_{\mathrm{ew}}[\mathrm{DN}, \mathrm{Lin}], \mathrm{FL}_{\mathrm{ew}}[\mathrm{DN}, \mathrm{Dis}]$ and $\mathrm{FL}_{\mathrm{ew}}[\mathrm{DN}]$, respectively.

(2) Gödel logic Int[Lin] is strictly stronger than Int. Thus, logics $\mathrm{FL}_{\text {ew }}[\mathrm{WCon}, \mathrm{Lin}]$ and $\mathrm{FL}_{\mathrm{ew}}[\mathrm{Lin}]$ are strictly stronger than $\mathrm{FL}_{e w}[\mathrm{WCon}, \mathrm{Dis}]$ and $F L_{\text {ew }}[\mathrm{Dis}]$, respectively.

We will show that $L$ in is not provable in $\mathrm{FL}_{\mathrm{ew}}[\mathrm{DN}, \mathrm{Dis}]$. Let $\mathrm{U}_{1}$ be the $\mathrm{FL}_{e w}$-algebra with the universe $\{0, \mathrm{~d}, \mathrm{c}, \mathrm{b}, \mathrm{a}, 1\}$ such that $0<\mathrm{d}<\mathrm{b}<\mathrm{a}<1$, $0<\mathrm{d}<\mathrm{c}<\mathrm{a}<1$ but $\mathrm{b}$ is incomparable with $\mathrm{c}$. Moreover, assume that $a^{2}=a, b^{2}=b, c^{2}=c, a \cdot b=b, a \cdot c=c, a \cdot d=0$ (thus, $x \cdot d=0$ if $x<a$ ) and $b \cdot c=0$. Then, it can be seen that $U_{1}$ is in fact a subdirectly irreducible, distributive $\mathrm{FL}_{e w}$-algebra satisfying $\sim \chi x=x$ for any $x$. Clearly, it is not linearly ordered. Thus, Lin is not valid in it.

(3) To see that $F L_{e w}[\mathrm{DN}, \mathrm{Lin}]$ is strictly weaker than $\mathrm{Cl}$, consider the $\mathrm{FL}_{e w^{-}}$ algebra $U_{2}$ with the universe $\{0, a, 1\}$ such that $0<a<1$ and $a^{2}=0$. Since $\sim a=a$ holds, $D N$ is valid but EM is not.

From this it follows that $\mathrm{FL}_{\mathrm{ew}}[\mathrm{Lin}]$ is strictly weaker than $\mathrm{FL}_{\text {ew }}[\mathrm{WCon}, \mathrm{Lin}]$. For, if WCon were provable in $\mathrm{FL}_{\mathrm{ew}}[\mathrm{Lin}]$ then it would be provable in $\mathrm{FL}_{\mathrm{ew}}[\mathrm{DN}, \mathrm{Lin}]$. Then, EM should be derived in $\mathrm{FL}_{\mathrm{ew}}$ [DN,Lin], since by Lemma 5.I it is provable in $\mathrm{FL}_{\mathrm{ew}}[\mathrm{WCon}, \mathrm{DN}]$. This is a contradiction. Similarly, we can show that $F L_{e w}[\mathrm{Dis}]$ and $F L_{e w}$ are strictly weaker than $\mathrm{FL}_{e w}$ [WCon, Dis] and $\mathrm{FL}_{\mathrm{ew}}$ [WCon], respectively.

(4) We will show next that Con is not provable in $\mathrm{FL}_{\mathrm{ew}}$ [WCon,Lin]. Let $\mathrm{U}_{3}$ be the $F L_{e w}$-algebra with the universe $\{0, b, a, 1\}$ such that $0<b<a<1$ and 
$b=a^{2}=a^{3}$. It is easy to see that $a \rightarrow b=a$ and $\sim a=\sim b=0$. It is clear that WCon is valid in a given $\mathrm{FL}_{e w}$-algebra if and only if $\sim x^{2}=\sim x$ for any $x$ in it. So, WCon is valid in $U_{3}$. On the other hand, Con is not valid in it, as $a \rightarrow a^{2}=a<1$. As a consequence, Con is not provable in $F L_{e w}$ [WCon], either.

(5) In [29], Grišin introduced a cut-free system for $\mathrm{FL}_{\mathrm{ew}}[\mathrm{DN}]$. Using this system, we can easily show that Dis is not provable in $\mathrm{FL}_{\mathrm{ew}}[\mathrm{DN}]$, and a fortiori is not provable in $\mathrm{FL}_{\mathrm{ew}}$. Now we show that Dis is not provable in $\mathrm{FL}_{\mathrm{ew}}$ [WCon]. Let $\mathrm{U}_{4}$ be the $\mathrm{FL}_{e w}$-algebra with the universe $\{0, d, c, b, a, 1\}$ such that $0<\mathrm{b}<$ $a<1,0<d<c<a<1$ and both $c$ and $d$ are incomparable with $b$. (Thus, $\mathrm{U}_{4}$ can be obtained from the nonmodular (and hence nondistributive) lattice $\mathrm{N}_{5}$, called the pentagon, by adding the new greatest element I. See e.g. [I.] Chapter 6.) Define also that $a^{2}=a, a \cdot b=b^{2}=b, a \cdot c=a \cdot d=c^{2}=c \cdot d=$ $d^{2}=d$ and $b \cdot c=b \cdot d=0$. Then, it can be seen that $U_{4}$ is a subdirectly irreducible, nondistributive $\mathrm{FL}_{e w}$-algebra. On the other hand, we can show that $\sim a=0, \sim b=c$ and $\sim c=\sim d=b$. Using this, we can show that WCon is valid in $\mathrm{U}_{4}$. Thus, Dis is not provable in $\mathrm{FL}_{\mathrm{ew}}$ [WCon].

\section{Simple and Semisimple FL $_{e w}$-Algebras}

In this section, we will discuss simple and semisimple $\mathrm{FL}_{e w}$-algebras. As usual, a simple $\mathrm{FL}_{e w}$-algebra $\mathrm{M}$ is defined to be a non-degenerate $\mathrm{FL}_{\mathrm{ew}}$-algebra which has only two filters $\{1\}$ and $M$ itself. It is easy to see that for any filter $F$ of a given $\mathrm{FL}_{e w}$-algebra $\mathrm{M}^{*}$ the quotient algebra $\mathrm{M}^{*} / \mathrm{F}$ is simple if and only if $\mathrm{F}$ is a maximal filter. Similarly to the case of Wajsberg algebras, the following holds. (See [14] Theorem 4.7 and [24] Corollary I of Theorem 17.)

LEMma 6.I An $\mathrm{FL}_{e w}$-algebra $\mathrm{M}$ is simple if and only iffor any $\mathrm{x}(<1)$ in $\mathrm{M}$ there exists a positive integer $\mathrm{m}$ such that $\mathrm{x}^{\mathrm{m}}=0$.

Clearly, any simple $\mathrm{FL}_{e w}$-algebra is subdirectly irreducible. It is well-known that for a Heyting algebra $M$, if it is simple then it is just the two-valued Boolean algbra $B_{2}$ and therefore satisfies that $\sim x=x$ for any $x$. On the other hand, this equation does not hold always in simple $\mathrm{FL}_{e w}$-algebras. Also, we know that any simple Wajsberg algebra is linearly ordered (see e.g. [I4, 4I, 24]). But, this does not hold either for $\mathrm{FL}_{e w}$-algebras, in general. In fact, we can give an example of a simple $\mathrm{FL}_{e w}$-algebra $\mathrm{N}$ which is not distributive (as a lattice) and hence is not linearly ordered. Let $\mathrm{M}_{3}$ be a nondistributive lattice, called the diamond (see e.g. [I9]). Let $\omega$ and o be the greatest element and the least element of $M_{3}$. Then $N$ is obtained from $M_{3}$ by adding a new element $I$ and requiring that $\omega<1$. Define operations and $\rightarrow$ on $N$ as follows; $x \cdot y=0$ for $x, y \in M_{3}$ and $x \cdot 1=1 \cdot x=x$ for any $x \in N$, and $x \rightarrow y=1$ if $x \leqslant y,=y$ if $x=1>y$ and $=\omega$ otherwise. Then, it is easily checked that $\mathrm{N}$ is a nondistributive, simple $\mathrm{FL}_{e w}$-algebra. 
Any $\mathrm{FL}_{e w}$-algebra $\mathrm{I}_{\mathrm{a}}$ for $0<a<1$ introduced in Section 4 is simple but does not have any coatom. Each Łukasiewicz's $n+1$-valued model $\succeq_{n+1}$ is a simple $F L_{e w}$-algebra, which is isomorphically represented as the $\mathrm{FL}_{e w}$-algebra with the set $\left\{1, a, a^{2}, \ldots, a^{n}\right\}$ such that $a^{i}>a^{i+1}$ for each $i=1, \ldots, n-1$ and $a^{n}=0$. For the simplicity's sake, let us denote $\mathrm{s}$ by $a^{0}$. Then the operation $\rightarrow$ on this $\mathrm{FL}_{e w}$-algebra can be defined by $\mathrm{a}^{\mathrm{k}} \rightarrow \mathrm{a}^{\mathrm{m}}=\mathrm{a}^{\max \{0, \mathrm{~m}-\mathrm{k}\}}$.

This representation might be convenient when we compare Łukasiewicz's models with other MV-algebras. For example, let us take the MV-algebra C discussed by Chang in [14], which is call $S_{1}{ }^{\omega}$ in Komori [4I]. Then, $C$ can be isomorphically represented as the residuated lattice with the set $\left\{1, a, a^{2}, \ldots, a^{n}, \ldots, \ldots, \sim a^{n}, \ldots, \sim a^{2}, \sim a, 0\right\}$ such that $a^{i-1}>a^{i}, a^{i}>\sim a^{j}$ and $\sim a^{j}>\sim a^{j-1}$ for each positive integer $i, j$. The operations $\cdot$ and $\rightarrow$ are defined as follows:

- $a^{k} \cdot a^{m}=a^{k+m}$,

$\cdot \mathrm{a}^{\mathrm{k}} \cdot\left(\sim \mathrm{a}^{\mathrm{m}}\right)=\sim \mathrm{a}^{\max \{\mathrm{m}-\mathrm{k}, 0\}}$,

- $\left(\sim \mathrm{a}^{\mathrm{k}}\right) \cdot\left(\sim \mathrm{a}^{\mathrm{m}}\right)=0$,

- $\mathrm{a}^{\mathrm{k}} \rightarrow \mathrm{a}^{\mathrm{m}}=\mathrm{a}^{\max \{0, \mathrm{~m}-\mathrm{k}\}}$,

- $\left(\sim a^{k}\right) \rightarrow a^{m}=1$,

- $\mathrm{a}^{\mathrm{k}} \rightarrow\left(\sim \mathrm{a}^{\mathrm{m}}\right)=\sim \mathrm{a}^{\mathrm{k}+\mathrm{m}}$,

- $\left(\sim \mathrm{a}^{\mathrm{k}}\right) \rightarrow\left(\sim \mathrm{a}^{\mathrm{m}}\right)=\mathrm{a}^{\max \{\mathrm{k}-\mathrm{m}, 0\}}$.

It is clear that $C$ is not simple and has only two proper filters $\{1\}$ and $\left\{a^{n}: n \in\right.$ $\mathrm{N}\}$.

For an $\mathrm{FL}_{e w}$-algebra $\mathrm{M}$, let $\Phi_{M}$ be the set of all maximal filters of $\mathrm{M}$. We note here that a filter $F$ is maximal if and only if for any $u \in M$ either $u \in F$ or

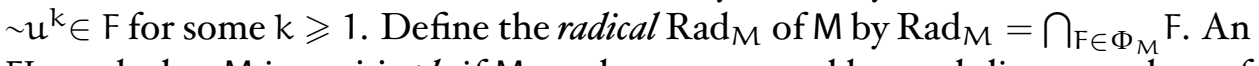
$\mathrm{FL}_{e w}$-algebra $\mathrm{M}$ is semisimple if $\mathrm{M}$ can be represented by a subdirect product of simple $\mathrm{FL}_{e w}$-algebras, or equivalently if $\operatorname{Rad}_{M}=\{1\}$ (see e.g. [48]). The proof of the following theorem is essentially due to Grišin [28]. In [29] he used the result to show that every free involutive $\mathrm{FL}_{e w}$-algebra is semisimple. From this, it follows that the logic $\mathrm{FL}_{\mathrm{ew}}[\mathrm{DN}]$ can be characterized by the class of simple involutive $\mathrm{FL}_{e} w$-algebras. Now, for any $x, y$ in a given $\mathrm{FL}_{e w}$-algebra, define $x+y$ by $x+y=\sim(\sim x \cdot \sim y)$. We can show easily that the operation + is associative and moreover that $(x+y)+z=\sim(\sim x \cdot \sim y \cdot \sim z)$. For an element $x$ and a positive integer $\mathrm{m}$, $\tilde{\mathrm{m}} x$ denotes $\sim(\sim x)^{\mathrm{m}}$. Then $\tilde{1} x=\sim \sim x$ and $\tilde{\mathfrak{m}} x=x+\cdots+x$ with $\mathrm{m}$ times $x$ when $m>1$.

THEOREM 6.I For any $x$ in a given $\mathrm{FL}_{\mathrm{ew}}$-algebra $\mathrm{M}, \mathrm{x} \in \operatorname{Rad}_{\mathrm{M}}$ if and only iffor any $\mathrm{n} \geqslant 1$ there exists $\mathrm{m} \geqslant 1$ such that $\tilde{\mathrm{m}}\left(\mathrm{x}^{\mathfrak{n}}\right)=1$. 
Proof: Suppose first that for any $n \geqslant 1$ there exists $m \geqslant 1$ such that $\tilde{m}\left(x^{n}\right)=1$. Suppose that $x \notin \operatorname{Rad}_{M}$. Then there exists a maximal filter $F$ such that $x \notin F$. Since $F$ is maximal, there exists a $k \geqslant 1$ such that $\sim x^{k} \in F$. there exists $m \geqslant 1$ such that $\tilde{\mathrm{m}}\left(x^{k}\right)=1$, i.e., $\left(\sim x^{k}\right)^{m}=0$. Thus, $0 \in \mathrm{F}$, which contradicts the fact that $F$ is proper.

Conversely, suppose that there exists $\mathfrak{n}(\geqslant 1)$ such that $\tilde{\mathfrak{m}}\left(x^{\mathfrak{n}}\right) \neq 1$ for any m. If $\left(\sim\left(x^{\mathfrak{n}}\right)\right)^{m}=0$ then $\tilde{\mathfrak{m}}\left(x^{\mathfrak{n}}\right)=\sim\left(\sim\left(x^{\mathfrak{n}}\right)\right)^{\mathrm{m}}=1$, which is a contradiction. Thus, $\left(\sim\left(x^{n}\right)\right)^{m}>0$ for any $m$. Let $z=\sim\left(x^{n}\right)$ and $H$ be the filter generated by $z$. Clearly, $\mathrm{H}$ is proper as $z^{\mathrm{m}}>0$ for any $\mathrm{m}$. By Zorn's lemma, there exists a maximal filter $G$ such that $H \subseteq G$. Now, suppose that $x \in G$. Then $x^{n}$ must be also in G. But this is a contradiction, since $z=\sim\left(x^{\mathfrak{n}}\right) \in \mathrm{G}$. Hence, $x \notin \mathrm{G}$ and therefore it does not belong to $\operatorname{Rad}_{M}$.

This result was also shown in Höhle [33]. As a corollary of Theorem 6.I. we have the following result on Wajberg algebras, which was shown by Font, Rodríguez and Torrens in [24]. (See also [33].)

COROLLARY 6.I For any $x$ in a given Wajsberg algebra $\mathrm{M}, \mathrm{x} \in \operatorname{Rad}_{\mathrm{M}}$ if and only if for any $\mathrm{n} \geqslant 1, \sim\left(\mathrm{x}^{\mathrm{n}}\right) \leqslant \mathrm{x}$.

Proof: By Theorem 6.I it suffices to show that in any Wajsberg algebra M, $\sim\left(x^{n}\right) \leqslant x$ for any $n \geqslant 1$ if and only if for any $n \geqslant 1$ there exists $m \geqslant 1$ such that $\tilde{m}\left(x^{\mathfrak{n}}\right)=1$.

Suppose first that $\sim\left(x^{n}\right) \leqslant x$. We will show that $\widetilde{n+1}\left(x^{n}\right)=1$. From the assumption, we have $\left(\sim\left(x^{n}\right)\right)^{n} \leqslant x^{n}$. Thus, $\sim x^{n} \leqslant \sim\left(\sim\left(x^{n}\right)\right)^{n}=\tilde{n}\left(x^{n}\right)$ and hence $1=\sim x^{n} \rightarrow \mathfrak{n}\left(x^{n}\right)=x^{n}+\tilde{n}\left(x^{n}\right)=\widetilde{n+1}\left(x^{n}\right)$. Conversely, suppose that $\sim\left(x^{n}\right) \not x$ for some $n \geqslant 1$. Take any subdirect representation $f: M \rightarrow \prod_{i} M_{i}$ with subdirectly irreducible factors $M_{i}$. Note that each $M_{i}$ is linearly ordered by Lemma 4.3 Let $p_{j}$ be the $j$-th projection function of the direct product $\prod_{i} M_{i}$, and let $z_{j}=\left(p_{j} \circ f\right)(x)$. By our assumption, $\sim\left(z_{j}\right)^{n} \nless z_{j}$ for some $j$. Since $M_{j}$ is linearly ordered, $z_{j} \leqslant \sim\left(z_{j}\right)^{n}$ holds. Therefore $\left(z_{j}\right)^{n+1}=0$. Then, $\tilde{\mathfrak{m}}\left(x^{\mathfrak{n}+1}\right)=1$ never hold for any $m$. This completes the proof of our lemma.

The next theorem gives us a sufficient condition for a $\mathrm{FL}_{e w}$-algebra to be semisimple. Here, we will introduce the following condition on $\mathrm{FL}_{e w}$-algebras:

$$
E M_{\mathrm{k}}: \chi \vee\left(\sim \chi^{\mathrm{k}}\right)=1 \text { for any } \chi .
$$

Note that $E M_{1}$ corresponds to an algebraic form of the excluded middle EM. THEOREM 6.2 The following three conditions are mutually equivalent for any subdirectly irreducible $\mathrm{FL}_{\text {ew }}$-algebra $\mathrm{M}$.

I. $\mathrm{M}$ is simple and satisfies $\mathrm{E}_{\mathrm{k}}$,

2. for each $x \in M$, if $x<1$ then $x^{k}=0$, 
3. Msatisfies $\mathrm{EM}_{\mathrm{k}}$.

Proof: Assume that $M$ is simple and satisfies $E M_{\mathrm{k}}$. If $\mathrm{x}<1$ then $1>x \geqslant x^{2} \geqslant$ $\cdots \geqslant x^{k}=x^{k+1}$. The filter $F$ generated by $x$ is expressed as $\left\{z \in M: x^{k} \leqslant z\right\}$. If $x^{k}>0$ then $F$ is neither equal to $\{1\}$ nor equal to $M$. But this contradicts our assumption that $\mathrm{M}$ is simple.

Next, suppose that $x<1$ implies $x^{k}=0$ for any $x \in M$. Take any $y \in M$. If $y=1$ then clearly $y \vee\left(\sim y^{k}\right)=1$. If $y<1, y^{k}=0$ by our assumption. Then, $\sim y^{k}=1$ and hence $y \vee\left(\sim y^{k}\right)=1$ holds also in this case.

Finally, suppose that $M$ satisfies $E M_{k}$. Suppose that $x<1$. Since $x \vee\left(\sim x^{k}\right)=$ $1, \sim x^{k}=1$ by Theorem 4.I. Then, $x^{k}=x^{k} \cdot 1=x^{k} \cdot \sim x^{k}=0$. Thus, $M$ is simple. Next, it is easy to see that for each $y, y^{k} \cdot y \leqslant y^{k+1}$ and $y^{k} \cdot \sim y^{k}=0 \leqslant y^{k+1}$. Therefore, $y^{k} \cdot\left(y \vee \sim y^{k}\right)=\left(y^{k} \cdot y\right) \vee\left(y^{k} \cdot \sim y^{k}\right) \leqslant y^{k+1}$. But, $y \vee \sim y^{k}=1$ by our assumption. Thus, $y^{k} \leqslant y^{k+1}$. Since the converse inequality holds always, we have that $y^{k}=y^{k+1}$ for any $y$.

Let us take I for $k$ in the above theorem. Then, we have the following wellknown result: For any subdirectly irreducible $\mathrm{FL}_{\mathrm{ew}}$-algebra $\mathrm{M}$, the following three conditions are mutually equivalent (cf. Lemma 5.I.).

I. $\mathrm{M}$ is simple and satisfies $\mathrm{x}^{2}=\mathrm{x}$ for any $\mathrm{x}$, i.e., $\mathrm{M}$ is a simple Heyting algebra,

2. $\mathrm{M}$ is isomorphic to the two-valued Boolean algebra $\mathrm{B}_{2}$,

3. M satisfies $\mathrm{x} \vee \sim \mathrm{x}=1$ for any $\mathrm{x}$.

A characterization of the variety defined by the condition $E M_{\mathrm{k}}$ and of the variety defined by $E M_{\mathrm{k}}$ from universal algebra is given in [43]. Following Chang [I4] and Komori [4I], for any $\mathrm{FL}_{e w}$-algebra $\mathrm{M}$, define the order $o(\mathrm{M})$ of $\mathrm{M}$ as follows: if $k$ is the smallest number $j$ such that $M$ satisfies $E M_{j}$, let $o(M)=k$, and if there exists no such $k$, let $o(M)=\omega$. For each $k$, let $\mathrm{EM}_{k}$ be the formula $p \vee \neg p^{k}$, which corresponds to the identity $E M_{k}$. It is obvious that for any $\mathrm{FL}_{e w}$-algebra $\mathrm{M}, o(\mathrm{M})=\mathrm{k}$ for a finite $k$ if and only if $k$ is the smallest number $j$ such that $\mathrm{FL}_{\mathrm{ew}}\left[\mathrm{EM}_{\mathrm{j}}\right] \subseteq \mathrm{L}(\mathrm{M})$.

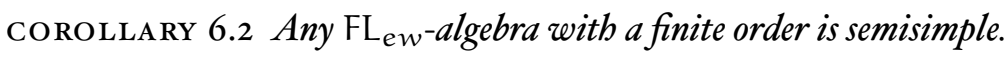

THEOREM 6.3 The intersection of $\mathrm{FL}_{e w}\left[E_{n}\right]$ for $\mathrm{n}<\omega$ is equal to $\mathrm{FL}_{e w}$.

Proof: Our theorem follows from the finite model property of $\mathrm{FL}_{\mathrm{ew}}$ proved by Okada and Terui in [50]. Suppose that a formula $A$ is not provable in $F L_{e w}$. Then by the finite model property of $F L_{e w}, A$ is not valid in a finite $F L_{e w^{-}}$ algebra $M$. Suppose that $M$ satisfies $E_{k}$. Then, $L(M)$ is a logic over $\mathrm{FL}_{e w}\left[\mathrm{E}_{k}\right]$ and thus, $A$ is not provable in $\mathrm{FL}_{\mathrm{ew}}\left[\mathrm{E}_{\mathrm{k}}\right]$.

In Kowalski and Ono [44], it is proved that the logic $\mathrm{FL}_{\mathrm{ew}}$ is determined by the class of finite, simple residuated lattices. This improves the result by Okada and Terui on the finite model property, and moreover implies immediately the following, using Theorem 6.2 . 
THEOREM 6.4 The intersection of $\mathrm{FL}_{\mathrm{ew}}\left[E M_{\mathrm{n}}\right]$ for $\mathrm{n}<\omega$ is equal to $\mathrm{FL}_{\mathrm{ew}}$.

Using the sequence $\left\{\mathrm{E}_{k}\right\}_{k}$, we introduce a classification $\left\{W_{k}\right\}_{k}$ of logics over $\mathrm{FL}_{\mathrm{ew}}$ in the following way.

- $\mathrm{W}_{1}=\left\{\mathrm{L}: \mathrm{FL}_{\mathrm{ew}}\left[\mathrm{E}_{1}\right] \subseteq \mathrm{L} \subseteq \mathrm{Cl}\right\}$

- $\mathrm{W}_{\mathrm{n}+1}=\left\{\mathrm{L}: \mathrm{FL}_{\mathrm{ew}}\left[\mathrm{E}_{\mathrm{n}+1}\right] \subseteq \mathrm{L} \subseteq \mathrm{Cl}\right\}-\mathrm{W}_{\mathrm{n}}$,

- $\mathrm{W}_{\omega}=\left\{\mathrm{L}: \mathrm{FL}_{\mathrm{ew}} \subseteq \mathrm{L} \subseteq \mathrm{Cl}\right\}-\bigcup_{\mathrm{n}<\omega} \mathrm{W}_{\mathrm{n}}$.

Clearly, the class $W_{1}$ is exactly the class of all intermediate logics. This classification is orthogonal to the class of Łukasiewicz's many-valued logics in the sense that for each $n>0$ only the logic $L\left(E_{n+1}\right)$ belongs to $W_{n}$ and that $L(€)$ belongs to $W_{\omega}$. By the definition of the classification, each $W_{n}$ has the smallest element $F L_{e w}\left[E_{n}\right]$ for each $0<n \leqslant \omega$, where $F L_{e w}\left[E_{\omega}\right]$ means $F L_{e w}$. On the other hand, $W_{n}$ has no greatest element in general, as shown in the next section.

\section{Neighbors of Classical logic}

A logic $\mathrm{L}$ over $\mathrm{FL}_{\mathrm{ew}}$ is an immediate predecessor of classical logic $\mathrm{Cl}$ if $(\mathrm{I}) \mathrm{L} \subset \mathrm{Cl}$ and (2) $\mathrm{L} \subseteq \mathrm{L}^{\prime} \subset \mathrm{Cl}$ implies $\mathrm{L}^{\prime}=\mathrm{L}$ for any logic $\mathrm{L}^{\prime}$. In this section, we will study immediate predecessors of $\mathrm{Cl}$. This, we hope, will be the first step in studying the whole structure of the lattice consisting of logics over $F L_{e w}$. ${ }^{\text {m }}$ The set of all immediate predecessors of $\mathrm{Cl}$ is denoted by $\mathrm{IP}(\mathrm{Cl})$ in the following.

Suppose that $\mathrm{L} \in \operatorname{IP}(\mathrm{Cl})$ and that $\mathrm{L}$ is characterized by an $\mathrm{FL}_{e}$-algebra $\mathrm{M}$. Let us assume moreover that $M$ is subdirectly represented by $\left\{N_{j}\right\}_{j \in J}$. Then, $\mathrm{L}(\mathrm{M}) \subseteq \mathrm{L}\left(\mathrm{N}_{j}\right) \subseteq \mathrm{Cl}$ for each $\mathrm{j} \in \mathrm{J}$. Since $\mathrm{L}(\mathrm{M})$ is an immediate predecessor of $\mathrm{Cl}$, some $L\left(N_{j}\right)$ must be equal to $L(M)$. Thus, we can assume that $L$ is characterized by a sudirectly irreducible $\mathrm{FL}_{\mathrm{ew}}$-algebra $\mathrm{M}$ from the beginning.

We have already seen some of immediate predecessors of $\mathrm{Cl}$. If we restrict our attention to logics over the intuitionistic logic Int, i.e. intermediate logics, then there exists the single immediate predecessor of $\mathrm{Cl}$, which is determined by the three valued Heyting algebra $\mathrm{H}_{3}$. In other words, the class $W_{1}$ has the single immediate predecessor $\mathrm{H}_{3}$ of $\mathrm{Cl}$. On the other hand, Komori [4I] discussed the lattice structure of logics over $\mathrm{L}(\mathrm{E})$ and proved that in this class $L$ is an immediate predecessor of $\mathrm{Cl}$ if and only if either $\mathrm{L}=\mathrm{L}\left(\mathrm{E}_{n+1}\right)$ with a prime number $n$ or $L=L(C)$, where $C$ is the MV-algebra of Chang (see the previous section). For each $n$ such that $0<n \leqslant \omega$, let $W_{n}{ }^{*}$ be the class of maximal elements in $W_{n}$, and $W_{n}{ }^{I P}$ be the set $W_{n} \cap \operatorname{IP}(C l)$, i.e. the set of all immediate predecessors of $\mathrm{Cl}$ in $W_{n}$. Clearly, $W_{n}{ }^{I P}$ is a subset of $W_{n}{ }^{*}$ when $n>1$.

\footnotetext{
${ }^{I I}(\dagger)$ For recent developments of the study in this direction, see Chapter 9 of $[25]$.
} 
THEOREM 7.I For each $\mathrm{n}<\omega, \mathrm{L}\left(\ell_{\mathrm{n}+1}\right)$ belongs to $\mathrm{W}_{\mathrm{n}}{ }^{*}$. Also, $\mathrm{L}(\mathrm{C})$ belongs to $\mathrm{W}_{\mathrm{\omega}}{ }^{*}$.

Proof: Suppose that $\mathrm{L}\left(\in_{n+1}\right) \subseteq \mathrm{L}$ and $\mathrm{L} \in W_{n}$. Clearly, $\mathrm{L}$ is a logic over $\mathrm{L}(\mathrm{E})$. Using results in Komori $[4 \mathrm{I}]$, there exists a finite set I of natural numbers such that $L=\bigcap_{i \in I} L\left(E_{i+1}\right)$. Then, by Lindenbaum's result, each $i \in I$ must be a divisor of $n$. If $i<n$ for all $i \in I$, then $L$ must be in $W_{j}$ where $j=\max \{i: i \in$ I $\}<n$. This is a contradiction. Thus, $i=n$ for some $i \in I$. Then, $L \subseteq L\left(E_{n+1}\right)$ and hence $L=L\left(E_{n+1}\right)$. Therefore, $L\left(E_{n+1}\right)$ is maximal in $W_{n}$. Since $L(C)$ belongs to $W_{\omega}{ }^{I P}$, the second part is obvious.

We will show in the rest of this section that there are many other immediate predecessors of $\mathrm{Cl}$. Note that each $\mathrm{E}_{n+1}$ is simple, but neither $\mathrm{H}_{3}$ nor $\mathrm{C}$ is so. It is clear that every $\mathrm{FL}_{e w}$-algebra $\mathrm{M}$ contains $\mathrm{M}$ itself and $\{0,1\}$ as its subalgebras. The latter is obviously isomorphic to the two-valued Boolean algebra $B_{2}$. A subalgebra $K$ of an $\mathrm{FL}_{e w}$-algebra $\mathrm{M}$ is trivial if $\mathrm{K}$ is either $\mathrm{M}$ or $\{0,1\}$. The following theorem gives us a condition for a finite subdirectly irreducible $\mathrm{FL}_{e w}$-algebra to determine an immediate predecessor of $\mathrm{Cl}$.

THEOREM 7.2 Suppose that $\mathrm{M}$ is a finite, subdirectly irreducible (non-degenerate) $\mathrm{FL}_{\mathrm{ew}}$-algebra. Then, $\mathrm{L}(\mathrm{M})$ is either equal to classical logic $\mathrm{Cl}$ or an immediate predecessor of $\mathrm{Cl}$ if and only if

I. every subalgebra of $\mathrm{M}$ is trivial,

2. for any proper filter $\mathrm{F}$ of $\mathrm{M}$, either $\mathrm{F}=\{1\}$ or the quotient algebra $\mathrm{M} / \mathrm{F}$ is isomorphic to $\mathrm{B}_{2}$.

To show Theorem 7.2, we use Jónsson's lemma [39] on congruencedistributive variety (see also [5]). Recall that the class of $\mathrm{FL}_{e w}$-algebras forms a congruence-distributive variety by Propositions 3.4 and 3.5 In the following, $\mathrm{V}(\mathcal{K})$ and $\mathrm{V}(\mathcal{K})_{\text {SI }}$ mean the variety generated by a class $\mathcal{K}$ of algebras and the class of subdirectly irreducible algebras in $\mathrm{V}(\mathcal{K})$, respectively. Also, $\mathrm{H}(\mathcal{K}), \mathrm{S}(\mathcal{K})$ and $\mathrm{Pu}_{\mathrm{u}}(\mathcal{K})$ denote the class of homomorphic images of members of $\mathcal{K}$, the class of subalgebras of members of $\mathcal{K}$, and the class of ultraproducts of nonempty families of members of $\mathcal{K}$, respectively $\check{L}^{2}$ We can state Jónsson's lemma applied to the present case as follows.

PROPOSITION 7.I Let $\mathcal{K}$ be a class of $\mathrm{FL}_{\text {ew }}$-algebras. Then, $\mathrm{V}(\mathcal{K})_{\mathrm{SI}} \subseteq \mathrm{HSP}(\mathcal{K})$. In particular, if $\mathrm{M}_{1}, \ldots, \mathrm{M}_{\mathrm{n}}$ are finite $\mathrm{FL}_{e w}$-algebras, then $\mathrm{V}\left(\left\{\mathrm{M}_{1}, \ldots, \mathrm{M}_{\mathrm{n}}\right\}\right)_{S \mathrm{I}} \subseteq$ $\mathrm{HS}\left(\left\{\mathrm{M}_{1}, \ldots, \mathrm{M}_{n}\right\}\right)$.

PROOF OF THEOREM 7.2. Only-if part is trivial. We assume that a finite subdirectly irreducible $M$ satisfies both conditions $I$ and 2. Moreover, suppose that $\mathrm{L}(\mathrm{M}) \subseteq \mathrm{L} \subset \mathrm{Cl}$ for a logic L. The logic $\mathrm{L}$ can be represented as $\bigcap_{i \in \mathrm{I}} \mathrm{L}\left(\mathrm{N}_{i}\right)$ for some non-degenerate, subdirectly irreducible $F L_{e w}$-algebras $N_{i}$ for $i \in I$. Since $\mathrm{L}(\mathrm{M}) \subseteq \mathrm{L}\left(\mathrm{N}_{i}\right), \mathrm{N}_{i} \in \mathrm{V}(\{\mathrm{M}\})_{S \mathrm{I}} \subseteq \mathrm{HS}(\{\mathrm{M}\})$, by using Proposition $7 . \mathrm{I}$ By

\footnotetext{
${ }^{\mathrm{I} 2}(\dagger)$ For more information on universal algebra, see [II].
} 
condition $\mathrm{I}, \mathrm{S}(\{\mathrm{M}\})=\left\{\mathrm{M}, \mathrm{B}_{2}\right\}$. Thus, each $\mathrm{N}_{i}$ must be the homomorphic image of some $\mathrm{K} \in\left\{\mathrm{M}, \mathrm{B}_{2}\right\}$ by a homomorphism $h$. Hence, the kernel $\operatorname{Ker}(\mathrm{h})$ of $\mathrm{h}$ is a proper filter of $\mathrm{K}$, and $\mathrm{N}_{\mathrm{i}} \cong \mathrm{K} / \operatorname{Ker}(\mathrm{h})$, by the homomorphism theorem. By condition 2, either $\operatorname{Ker}(h)=\{1\}$ or the quotient algebra $N_{i}$ is isomorphic to $B_{2}$. In other words, $N_{i}$ is isomorphic either to $K \in\left\{M, B_{2}\right\}$ or to $B_{2}$. Since $L=\bigcap_{i \in I} L\left(N_{i}\right) \subset C l, N_{j}$ must be isomorphic to $M$ for some $j \in I$. Therefore, $\mathrm{L}=\mathrm{L}(\mathrm{M})$ as $\mathrm{L}(\mathrm{M}) \subseteq \mathrm{L}\left(\mathrm{B}_{2}\right)$. Thus, $\mathrm{L}(\mathrm{M})$ is an immediate predecessor of $\mathrm{Cl}$.

COROLLARY 7.I If $\mathrm{M}$ is a finite, simple (non-degenerate) $\mathrm{FL}_{\text {ew-algebra without non- }}$ trivial subalgebras, then $\mathrm{L}(\mathrm{M})$ is equal either to classical logic $\mathrm{Cl}$ or to an immediate predecessor of $\mathrm{Cl}$.

Proof: If $\mathrm{M}$ is simple, it has only $\{1\}$ as its proper filter. Thus, condition 2 in Theorem 7.2 is satisfied.

This corollary gives us a proof of a well-known result which says that $\mathrm{L}\left(\mathrm{E}_{n+1}\right)$ is an immediate predecessor of $\mathrm{Cl}$ when $\mathrm{n}$ is prime. Note that in [6I] K. Swirydowicz showed the existence of infinitely many immediate predecessors of $\mathrm{Cl}$ over the classical linear logic, i.e. $\mathrm{FL}_{e}[\mathrm{DN}]$, by using the similar criterion as Corollary 7.I.

By Theorem 6.2 and Corollary 6.2, we have the following.

COROLlaRY 7.2 Suppose that an immediate predecessor $\mathrm{L}$ of $\mathrm{Cl}$ is determined by an

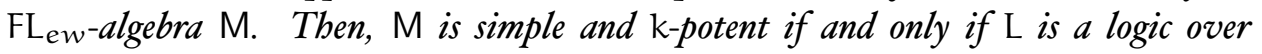
$\mathrm{FL}_{\mathrm{ew}}\left[E M_{\mathrm{k}}\right]$.

By using Corollary 7.I, we will give here an example of simple, linearly ordered $\mathrm{FL}_{e w}$-algebra $\mathrm{K}$ which determines an immediate predecessor of $\mathrm{Cl}$, but which is not isomorphic to any of Łukasiewicz's models. The universe of $\mathrm{K}$ consists of 6 elements, which are ordered as $1>a>b>a^{2}>a^{3}>a^{4}=0$. We assume that $a \cdot b=b^{2}=a^{3}$. Then, from this it follows that $a^{2} \cdot b=b^{3}=0$. Moreover, (nontrivial) implications are determined as follows. $a \rightarrow b=b \rightarrow$ $a^{2}=b \rightarrow a^{3}=a, \sim b=a^{2}$, and for $1 \leqslant m<n \leqslant 4, a^{m} \rightarrow a^{n}=a^{n-m}$ if $n-m \neq 2$, and $=b$ otherwise. It is easy to see that $K$ has no nontrivial subalgebras. Thus, it determines an immediate predecessor of $\mathrm{Cl}$ which belongs to $\mathrm{W}_{4}$.

It will be interesting to see whether there is an immediate predecessor of $\mathrm{Cl}$ or not, which is determined by a simple nonlinear $\mathrm{FL}_{e w}$-algebra, or by a simple infinite $\mathrm{FL}_{e w}$-algebra.

In the rest of this section, we will discuss such subdirectly irreducible, nonsimple $\mathrm{FL}_{\mathrm{ew}}$-algebras that determine immediate predecessors of $\mathrm{Cl}$. First, as an application of Theorem 7.2 we will give a series $\left\{\mathrm{J}_{\mathrm{n}}\right\}_{\mathrm{n}}$ of nonsimple and nonlinear $\mathrm{FL}_{e w}$-algebras such that any of them determines an immediate predecessor of $\mathrm{Cl}$. (The same result is obtained independently by T. Kowalski.) For each $n>1$, the universe of $J_{n}$ consists of $n+3$ elements $\left\{1, a, a^{2}, \ldots, a^{n}(=\right.$ $\left.\left.a^{n+1}\right), b, 0\right\}$. The element $b$ satisfies $a^{n-1}>b>0$ and is incomparable with 
$a^{n}$. We assume moreover that $a \cdot b=0$, and thus $x \cdot b=0$ for any element $x \leqslant a$. Then, the following holds:

I. $a^{k} \rightarrow a^{m}=a^{m-k}$ if $k<m$,

2. $a^{k} \rightarrow b=\sim a^{k}=b$ for $1 \leqslant k \leqslant n$,

3. $b \rightarrow a^{n}=\sim b=a$.

Clearly, the element $a$ generates $J_{n}$ since $\sim a=b$. Also, $b$ generates it as $\sim b=a$. Finally, each $a^{k}$ for $1<k \leqslant n$ generates it, since $\sim a^{k}=b$. Thus, $J_{\mathfrak{n}}$ has no nontrivial subalgebras. The algebra $J_{\mathfrak{n}}$ has the single proper filter $F=\left\{1, a, a^{2}, \ldots, a^{n}\right\}$ except $\{1\}$. It is obvious that the quotient algebra $J_{\mathfrak{n}} / F$ is isomorphic to $B_{2}$. Thus, by Theorem 7.2 each $J_{n}$ determines an immediate predecessor of $\mathrm{Cl}$ in $W_{n}$. Hence, we have the following ${ }^{13}$

THEOREM 7.3 For each $n>1, W_{n}$ contains an immediate predecessor of $\mathrm{Cl}$, which is determined by the nonsimple and nonlinear $\mathrm{FL}_{e w}$-algebra $\mathrm{J}_{\mathrm{n}}$.

In the following, we will assume that $M$ is a subdirectly irreducible, nonsimple $\mathrm{FL}_{\mathrm{ew}}$-algebra satisfying $E_{\mathrm{n}}$ for some $\mathrm{n}>1$, which moreover determines an immediate predecessor of $\mathrm{Cl}$. From Corollary 4.2 it follows that $\mathrm{M}$ has the unique coatom $a$. When $\mathrm{a}=0, \mathrm{M}$ becomes the 2 -valued Boolean algebra, and therefore it is simple, which is a contradiction. Hence, we have that $a>0$ and $a^{n}=a^{n+1}>0$. Let $b=a^{n}$. Then, the filter $F$ generated by $a$ can be represented as $\{x: b \leqslant x\}$, which is a proper filter, not equal to $\{1\}$. Therefore, by Theorem 7.2 the quotient algebra $M / F$ must be isomorphic to $B_{2}$. In particular, $\|b\|=\|1\|$ and $\sim\|b\|=\|0\|$ hold, where $\|x\|$ denotes the equivalence class to which an element $x$ belongs. Thus, $b \nless \sim b$. Hence, either $\sim \mathrm{b}<\mathrm{b}$ or $b$ is incomparable with $\sim b$. We assume that the former holds, and consider moroever two special cases; (I) $\sim b=0$, and (2) $\sim b>0$ and $\sim b=b$.

I. Case where $\sim b=0$ holds: Since $b^{2}=b$, the set $\{0, b, 1\}$ forms a subalgebra of $M$, which is isomorphic to the three valued Heyting algebra $\mathrm{H}_{3}$. (Note that when $M$ is a Heyting algebra, both $b=a$ and $0=\sim b<b$ hold always.) Thus, $M$ itself must be isomorphic to $\mathrm{H}_{3}$, as it does not contain nontrivial subalgebras.

${ }^{13}$ After the original draft of the present paper is completed, in his master thesis M. Ueda obtained a far stronger result than our Theorem 7.3 which say that for each $n>1, W_{n}$ contains countably many immediate predecessors of $\mathrm{Cl}$, each of which is determined by a nonsimple and nonlinear $\mathrm{FL}_{\mathrm{ew}}$-algebra, and that for each $\mathrm{n}>2, \mathrm{~W}_{n}$ contains also countably many immediate predecessors of $\mathrm{Cl}$, each of which is determined by a nonsimple and linear $\mathrm{FL}_{e w}$-algebra. Then, T. Kowalski improved them and showed that "countably many" can be replaced by "uncountably many" in them. As Ueda and Kowalski have obtained other stronger results, we have now a much clearer picture of immediate predecessors of $\mathrm{Cl}$ and the rest of the present paper should be revised. But, as we mentioned at the beginning, we decided not to make much revisions. For more information on Ueda and Kowalski's results, see $[56$, and $[40]$. 
2. Case where both $b>\sim b>0$ and $\sim b=b$ hold: It is easily seen that $(\sim b)^{2}=0$ and $b \rightarrow \sim b=\sim b$ hold. Thus, the set $\{0, \sim b, b, 1\}$ forms $a$ subalgebra of $M$, which we call $I_{1}$. Hence, $M$ must be isomorphic to $I_{1}$.

From these observations, the following result follows.

THEOREM 7.4 Suppose that $\mathrm{M}$ is a subdirectly irreducible, nonsimple $\mathrm{FL}_{\mathrm{ew}}$-algebra satisfying $E_{\mathrm{n}}$ for some $\mathrm{n}>1$ and moreover that $\mathrm{L}(\mathrm{M})$ is an immediate predecessor of $\mathrm{Cl}$.

I. If $\mathrm{M}$ satisfies the axiom $\neg p \vee \neg \neg p$ then $\mathrm{M}$ is isomorphic to $\mathrm{H}_{3}$.

2. If $\mathrm{M}$ satisfies both DN and the axiom $(\mathrm{p} \rightarrow \neg \mathrm{p}) \vee(\neg p \rightarrow p)$ then $\mathrm{M}$ is isomorphic to $\mathrm{I}_{1}$.

Proof: Take elements $a$ and $b$ just in the same way as in the above.

I). Since $\sim \mathfrak{b} \vee \sim \sim b=1$, either $\sim b=1$ or $\sim \sim b=1$ holds. If the former is the case, then $a^{n}=b=0$. This contradicts our assumption that $M$ is not simple. Thus, $\sim \mathrm{b}=1$ holds and hence $\sim \mathrm{b}=0$ holds. Then, by the above (I) $\mathrm{M}$ is isomorphic to $\mathrm{H}_{3}$.

2). In this case, either $b \leqslant \sim b$ or $\sim b<b$ holds. But the former does not hold as shown in the above. Since $\sim \mathrm{b}=\mathrm{b}<1$ must hold, $\sim \mathrm{b} \neq 0$. Thus, $\mathrm{M}$ is isomorphic to $I_{1}$ by (2).

Note that the formula $(\neg p \vee \neg \neg p) \rightarrow((p \rightarrow \neg p) \vee(\neg p \rightarrow p))$ is provable in $\mathrm{FL}_{\text {ew }}$. At present, we do not have any clear view of immediate predecessors of $\mathrm{Cl}$ yet, even if we restrict attention only to $W_{2}{ }^{I P}$. It is easy to see that $\mathrm{L}\left(\mathrm{E}_{3}\right)$ is the single member of $W_{2}{ }^{I P}$ which is determined by a simple $\mathrm{FL}_{e w}$-algebra. The class $W_{2}{ }^{I P}$ contains $L\left(I_{1}\right), L\left(J_{2}\right)$ and more. In fact, in 2000 T. Kowalski and $M$. Ueda proved that $W_{n}{ }^{I P}$ contains uncountably many logics (see [40] for the details).

\section{REFERENCES}

[I] Adillon, R.J., and v. verdú, On product logic, Soft Computing 2, (I998) pp. I4I-I46.

[2] agliano, P., i.m.A. FERreirim and F. MONTAgna, Basic hoops: an algebraic study of continuous t-norms, Studia Logica 87, (2007) pp. 73-98.

[3] bAAZ, M., P. HÁJEK, J. KRAJíčEK and D. šVEJdA, Embedding logics into product logic, Studia Logica 6I, (1998) pp. 35-47.

[4] Balbes, R. and PH. Dw inger, Distributive Lattices, Univ. Missouri Press, I974.

[5] вLOK, w.J., The lattice of modal logics: An algebraic investigation, fournal of Symbolic Logic 45, (1980) pp. 22I-236. 
[6] Blok, W.J. and i.m.A. Ferreirim, Hoops and their implicational reducts (abstract), Algebraic Methods in Logic and Computer Science, Banach Center Publications 28, (1993) pp. 219-230.

[7] blok, w.J. and i.m.A. Ferreirim, On the structure of hoops, Algebra Univerisalis 43 , (1999) pp. 233-257.

[8] вцок, w.J. and D. PIGOzzi, On the structure of varieties with equationally definable principal congruence III, Algebra Universalis 32, (I994) pp. 545-608.

[9] BLOK, W.J. and J.G. RAFTERY, On the quasivariety of BCK-algebras and its subvarieties, Algebra Univerisalis 33, (1995) pp. 68-90.

[IO] BLOK, w.J. and J.G. RAFTERY, Varieties of commutative residuated integral pomonoids and their residuation subreducts, fournal of Algebra 190 , (1997) pp. 280-328.

[ir] burris, s. and H.p. sankappanavar, $A$ Course in Universal Algebra, Graduate Texts in Mathematics, Springer, 198I, available on line.

[I2] CASARI, E., Conjoining and disjoining on different levels, Logic and Scientific Methods, eds. by M.L. Dalla Chiara et al., Kluwer Academic Publishers, I997, pp. 26I-288.

[i3] Chagrov, a.v. and M. v. Zakharyaschev, Modal Logic, Oxford University Press, 1997 .

[I4] ChANG, C.C., Algebraic analysis of many-valued logics, Transactions of the American Mathematical Society 88, (1958) pp. 467-490.

[15] CHANG, C.C., A new proof of the completeness of the Eukasiewicz axioms, Transactions of the American Mathematical Society 93, (1959) pp. 74-8o.

[i6] Cignoli, R., i.m.L. D'ottaviano and D. Mundici, Algebraic Foundations of Many-valued Reasoning, Studia Logica Library, Kluwer Academic Publishers, 2000.

[I7] Cignoli, R., F. esteva, L. GOdo and A. torrens, Basic fuzzy logic is the logic of continuous t-norms and their residua, Soft Computing 4, (2000) pp. IO6-II2.

[18] CORnish, w.H., Varieties generated by finite BCK-algebras, Bulletin of Australian Mathematical Society 22, (I980) pp.4II-43O.

[19] Davey, B.A. and H.A. PRiestley, Introduction to Lattices and Order 2nd edition, Cambridge University Press, Cambridge, 2002. 
[20] DilwORTH, R.P., Non-commutative residuated lattices, Transactions of the American Mathematical Society 46, (1939) pp. 426-444.

[2I] Došen, K. and P. Schroeder-heister eds., Substructural logics, Oxford Univ. Press, 1993.

[22] DošEn, K., Sequent systems and groupoid models. I, Studia Logica 47, (1988) pp. 353-385.

[23] Došen, K., Sequent systems and groupoid models. II, Studia Logica 48, (I989) pp. 4I-65.

[24] FONT, J.M., A.J. RODRÍGUez and A. TORRENS, Wajsberg algebras, Stochastica 8, (1984) pp. 5-3I.

[25] Galatos, N., P. JipSen, T. Kowalsk i and H. ONo, Residuated Lattices: an Algebraic Glimpse at Substructural Logics, Studies in Logic and the Foundations of Mathematics I5I, Elsevier, 2007.

[26] Girard, J.Y., Linear logic, Theoretical Computer Science 5o, (I987) pp. IIO2.

[27] GRIŠIN, V.N., On a nonstandard logic and its application to the set theory (in Russian), Isslédovanija po formalizovannym jazykam i néklassičéskim logikam, Moskva, 1974, pp. 135-17I.

[28] GRIŠ IN, V.N., On algebraic semantics for logic without the contraction rule (in Russian), Isslédovanija po formalizovannym jazykam i néklassičéskim logikam, Moskva, I976, pp. 247-264.

[29] GRIŠ In, V.N., Predicate and set-theoretical calculi based on logic without the contraction rule (in Russian), Izvéstia Akademii Nauk SSSR 45, (198I) pp. 47-68 (also the English translation in Math. USSR Izvestiya I8, (1982) pp. 4I-59).

[30] нÁje K, P., Metamathematics of Fuzzy Logic, Trends in Logic 4, Studia Logica Library, Kluwer Academic Publishers, 1998.

[3I] HÁJEK, P., L. GODO and F. ESTEVA, A complete many-valued logic with product-conjunction, Archive for Mathematical Logic 35, (1996) pp. I9I208.

[32] HIGGS, D., Residuated commutative monoids with identity element as least element do not form an equational class, Mathematica faponica 29, (I984) pp. 69-75.

[33] нӧнle, U., Commutative residuated monoids, Non-classical Logics and Their Applications to Fuzzy Subsets eds. by U. Höhle and P. Klement, Dordrecht, Kluwer, I995, pp. 53-106. 
[34] IDZIAK, P.M., Lattice operation in BCK-algebras, Mathematica faponica 29, (1984) pp. 839-846.

[35] Iśeki, K., BCK-algebras with condition (S), Mathematica faponica 24, (I979) pp. IO7-II9.

[36] iśeki, K. and s. TANaka, An introduction to the theory of BCKalgebras, Mathematica faponica 23, (1978) pp. I-26.

[37] JANkov, v.A., On connection between provability in intuitionistic propositional calculus and finite implicative structures (in Russian), Doklady Akademii Nauk SSSR, I5I, (1963) pp. 1293-1294.

[38] johnstone, в.т., Conditions related to de Morgan's law, Applications of Sheaves, Lecture Notes in Mathematics 753, Springer, 1979, pp. 479-49I.

[39] Jónsson, B., Algebras whose congruece lattices are distributive, Mathematica Scandinavica 2I, (1967) pp. IIO-I2I.

[40] катон, Ү., т. KOWALSK I and M. UeDA, Almost minimal varieties related to fuzzy logics, Reports on Mathematical Logic 4I, (2006) pp. I73-I94.

[4I] комоR I, Y., Super-Łukasiewicz propositional logics, Nagoya Mathematical fournal 84, (I98I) pp. II9-I33.

[42] kowalski, т., The bottom of the lattice of BCK-varieties, Reports on Mathematical Logic 29, (1995) pp. 87-93.

[43] KOWALSK I, T., Semisimplicity, EDPC and discriminator varieties of residuated lattices, Studia Logica 77, (2005) pp. 255-265.

[44] KOWALS к I, т. and н. ONO, The variety of residuated lattices is generated by its finite simple members, Reports on Mathematical Logic 34, (2000) pp. $57-75$.

[45] кOWALSк I, т. and н. ONO, Splittings in the variety of residuated lattices, Algebra Universalis 44, (2000) pp. 283-298.

[46] Kowals к I, т. and H. ONo, Residuated Lattices: An algebraic glimpse at logics without contraction (preliminary report), March, 2OOI.

[47] Krull, w., Axiomatische Begründung der allgemeinen Idealtheorie, Sitzungsberichte der physikalisch medizinischen Societät der Erlangen 56, (1924) pp. 47-63.

[48] mCKenzie, R.N., G.F. mCNUlty and w.F. TAilor, Algebras, Lattices, Varieties vol.., Wadsworth \& Brooks/Cole, California, 1987.

[49] MUNDICI, D., MV-algebras are categorically equivalent to bounded commutative BCK-algebras, Mathematica Faponica 3I, (1986) pp. 889-894. 
[50] OKADA, M. and K. TERUI, The finite model property for various fragments of intuitionistic linear logic, Journal of Symbolic Logic, (1999) pp. $790-802$.

[5I] ONO, H., Some remarks on semantics for the classical logic without the contraction rules, Reports on Mathematical Logic 19, (1985) pp. 3-12.

[52] ONO, H., Structual rules and a logical hierarchy, Mathematical Logic, edited by P.P. PET Kov, Plenum Press, 1990, pp. 95-IO4.

[53] ONO, H., Semantics for substructural logics, Substructural Logics, eds. by K. Došen and P. Schroeder-Heister, Oxford Univ. Press, I993, pp. 25929I.

[54] ONO, H., Proof-theoretic methods in nonclassical logic - an introduction, Theories of Types and Proofs, MSJ Memoir 2, eds. by M. Takahashi, M. Okada and M. Dezani-Ciancaglini, Mathematical Society of Japan, I998, pp. 207-254.

[55] ONO, H. and Y. KOMOR I, Logics without the contraction rule, fournal of Symbolic Logic 50 (1985) pp. I69-20I.

[56] ONO, H. and M. UEDA, A classification of logics over $\mathrm{FL}_{\mathrm{ew}}$ and almost maximal logics, Philosophical Dimensions of Logic and Science, eds. by A. Rojszczak, J. Cachro and G. Kurczewski, Kluwer, 2003, pp.3-13.

[57] PAŁASIŃSKI, M., Subdirectly irreducibles in $E_{n}$-varieties of BCKalgebras, preprint.

[58] paVelka, J., On fuzzy logic in, Enriched residuated lattices and semantics of propositional calculi, Zeitschrift für der mathematische Logik und Grundlagen der Mathematik 25, (1979) pp. II9-I34.

[59] rosenthal, K.I., Quantales and Their Applications, Pitman Research Notes in Mathematics 234, Longman, 1990.

[6o] SChwe Izer, B. and A. S KLAR, Probabilistic Metric Spaces, North-Holland, 1983.

[6r] Swiry DOwICZ, K., There exist infinitely many Post-complete extensions of the classical linear logic, manuscript, 1996.

[62] WARD, M., Residuated distributive lattices, Duke Mathematical fournal 6, (I940) pp. 64I-65I.

[63] Ward, M. and R.P. Dilworth, Residuated lattices, Transactions of the American Mathematical Society 45, (1939) pp. 335-354. 
[64] Wrońs k I, A., BCK-algebras do not form a variety, Mathematica faponica 28 , (I983) pp. 2II-2I3.

[65] wrońsкi, A., An algebraic motivation for BCK-algebras, Mathematica Faponica 3o, (1985) pp. 187-193.

[66] yutani, H., On a system of axioms of commutative BCK-algebras, Mathematics Seminar Notes of the Kobe University 5, (1977) pp. 255-256.

[67] zlatoš, P., Two-levelled logic and model theory, Colloquia Mathematica Societatis Fános Bolyai 28, Finite Algebra and Multiple-Valued Logic, Szeged, I979, pp. 825-872. 
The Australasian fournal of Logic (ISSN I448-5052) disseminates articles that significantly advance the study of logic, in its mathematical, philosophical or computational guises. The scope of the journal includes all areas of logic, both pure and applied to topics in philosophy, mathematics, computation, linguistics and the other sciences.

Articles appearing in the journal have been carefully and critically refereed under the responsibility of members of the Editorial Board. Only papers judged to be both significant and excellent are accepted for publication.

The journal is freely available at the journal website at

$$
\text { http://www.philosophy.unimelb.edu.au/ajl/ }
$$

All issues of the journal are archived electronically at the journal website.

SuBSCRIPTIONS Individuals may subscribe to the journal by sending an email, including a full name, an institutional affiliation and an email address to the managing editor at ajl-editors@unimelb.edu.au Subscribers will receive email abstracts of accepted papers to an address of their choice. For institutional subscription, please email the managing editor at ajl-editors@unimelb.edu.au.

Complete published papers may be downloaded at the journal's website at http: //www.philosophy.unimelb.edu.au/ajl/ The journal currently publishes in pdf format.

Submission The journal accepts submissions of papers electronically. To submit an article for publication, send the $\mathrm{LT}_{\mathrm{E}} \mathrm{X}$ source of a submission to a member of the editorial board. For a current list of the editorial board, consult the website.

The copyright of each article remains with the author or authors of that article. 